\title{
Multilinear target-based decision analysis with hybrid-information targets and performance levels
}

\author{
Xinwei Zhang ${ }^{1}(1) \cdot$ Qiong Feng $^{1}\left([) \cdot\right.$ Shurong Tong $^{1} \cdot$ Hakki Eres $^{2}$
}

Accepted: 25 November 2021 / Published online: 30 January 2022

(c) The Author(s), under exclusive licence to Springer Science+Business Media, LLC, part of Springer Nature 2022

\begin{abstract}
For several classes of decisions, the use of target-based decision analysis (TBDA) is more appropriate than utility analysis. Recent literature on TBDA mainly focuses on different expression forms of targets and performance levels, and on different types of aggregation operators in terms of multi-attribute preference functions. However, different expression forms are usually introduced separately in literature, which cannot fully support the inherent complexity of problems along with different perception and knowledge levels of decision makers during assessing targets and performance levels. Furthermore, although there are attractive features of multilinear target-based preference functions (MTPFs), its applications are seldom considered because of the complexity of identifying their coefficients. In order to solve these two issues, an integrated approach is proposed for multilinear hybrid-information target-based decision analysis, which can deal with diverse forms of targets and performance levels, and identify the coefficients of MTPFs. First, given targets and performance levels for each attribute being expressed in multiple forms simultaneously, a generalized procedure is proposed to transform different forms into probability distributions, and to measure probability of target achievement for each attribute. Second, a novel procedure to identify the coefficients of MTPFs is proposed. This procedure is based on the multilinear model and 2-additive fuzzy measures, which is based on the equivalence between multilinear model based on fuzzy measures and MTPFs. The approach is applied to a
\end{abstract}

Qiong Feng

fq@mail.nwpu.edu.cn

Xinwei Zhang

xwzhang7889@nwpu.edu.cn

Shurong Tong

stong@nwpu.edu.cn

Hakki Eres

M.H.Eres@soton.ac.uk

1 School of Management, Northwestern Polytechnical University, Xi' an, China

2 Faculty of Engineering and Physical Sciences, University of Southampton, Southampton, UK 
case study involving customer competitive evaluation of smart thermometer patches to demonstrate its feasibility and advantages.

Keywords Target-based decision analysis - Multilinear target-based preference functions · Hybrid-information expressions · Multilinear model · 2-additive fuzzy meausres

\section{Introduction}

Targets, or aspirations, appear widely and play an important role in many situations. They can be related to a product, such as targets on weight and performance, or an organization, such as target on profit and market share. They can be certain, as in the case of a target on profit defined by a crisp number, or random, as in the case of a product's attribute target coming from competing products under development, or fuzzy, as in the case of a target expressed by linguistic expressions. They can involve a single attribute or multiple attributes (e.g., product alternative evaluation or provider evaluation based on a set of attributes). There is ample empirical evidence that targets play an important role in many decisions. According to Simon's satisficing model, targets can simplify the cognitive processes of decision-making. As indicated in Bordley and Kirkwood (2004); Tsetlin \& Winkler, 2006), target and target-based decision making are more natural than utility and utility-based decision making in several situations, such as product development, regulated environments, setting performance standards, and resource allocation under uncertain competition.

Compared to utility theory, target-based decision analysis (TBDA) interprets the NM-utility function for a single attribute as a cumulative distribution function (CDF) of an uncertain target (Bordley \& LiCalzi, 2000), that is a target achievement function. In other words, when a decision maker is target-oriented, the utility may not depend on an absolute performance level, but rather on whether the performance level achieves a target. If the target is certainly achieved or not achieved, target achievement function can be a binary utility function. If the target and target achievement is uncertain, TBDA prescribes that the decision maker should choose an alternative which maximizes the probability of achieving the uncertain target in case of a single attribute (Bordley \& Kirkwood, 2004). Accordingly, it is an important process in TBDA to construct target-based preference functions to legitimately obtain overall target achievement probabilities of alternatives.

TBDA has attracted the attention of some scholars. Borch (1968) introduces the concept of target-based utility, using a different terminology of probability of ruin. Berhold (1973) notes that the use of CDFs to represent utility functions can take advantage of the various properties and existing results of these CDFs. Li Calzi et al. (1996, 2000) prove that expected utility model can be expressed in the language of probability based on von Neumann and Morgenstern's axiomatization and Savage's axiom. Huynh et al. (2007) develop a fuzzy target-based approach for decision making uncertainty when there is only one payoff attribute. With regard to multiple attributes, Bordley and Kirkwood (2004) propose an approach based on targets to assess three specific classes of multi-attribute target-based preference functions, which are proved 
to be strategically equivalent to the corresponding multi-attribute utility functions. Different from the case of single attribute, Tsetlin and Winkler (2006) show that a multi-attribute utility function cannot always be expressed in the form of a CDF and cannot always be expressed in the form of a target-based utility function. From a more general context, they study the impact on expected utility by modifying parameters of performance distribution or target distribution when targets and performance levels are dependent (Tsetlin \& Winkler, 2007). Spizzichino et al. (2019) point out the connection between multi-attribute target-based utilities and copulas-based extensions of fuzzy measures that are the generalization of the multilinear model and Choquet integral. Moreover, some aggregation operators are applied to construct TBDA model, such as weighted aggregating operator (Wang et al., 2019), Choquet integral (Yan et al., 2013) and so on.

About the extensions and applications of TBDA in specific fields and problems, Yan et al. (2008) apply fuzzy TBDA to quantify how well a product achieves consumers' preference and a prioritized scoring aggregation operator is utilized to aggregate probability of single-attribute target achievement. Feng and Lai (2014) introduce a multi-attribute group decision making approach with targets for strategic freight forwarder selection. Huynh et al. (2010) propose a linguistic TBDA approach to consumer-oriented Kansei evaluation and Wang et al. (2019) propose a TBDA approach for qualitative decision-making with complex linguistic expressions and apply it to a provider selection problem. TBDA has also been applied to support optimal resource allocation in transportation security (Kosanoglu \& Bierb, 2020).

The current research has made significant contributions to TBDA. The equivalence between the NM-utility and the target-based utility has been recognized and formally proved for the case of single attribute. Different forms of targets and performance levels, such as random, fuzzy and linguistic forms, have been analyzed for calculating probability of target achievement. With regard to multiple attributes, specific classes of multi-attribute target-based preference functions have been established for certain independent conditions, such as additive form, multiplicative form and multilinear form.

However, the current research still has two limitations. The first limitation is that specific forms of targets and performance levels are usually introduced separately in literature, which further requires diverse forms of targets and performance levels to be synthesized into a unified framework. Due to the complexity of practical problems and different levels of cognition and knowledge of decision makers, targets and performance levels of different attributes are possibly assessed in diverse forms at the same time, such as crisp numbers, interval numbers, fuzzy numbers, probability distributions, simple linguistic terms and complex linguistic expressions. Practically, the decision makers should be given freedom to assess fruitful and flexible forms to enable effective evaluation, and these diverse forms of expression should be dealt with in a unified framework. The second limitation is that applications of multi-attribute targetbased preference functions mainly focus on additive forms (Bordley \& Kirkwood, 2004; Wang et al., 2019), simplifying the relationships among attributes in aggregation functions. Additive target-based preference functions require additive target preferences to be verified. That is, on basis of independent target, additive independent preferences are also necessary. Additive target preferences are a strong assumption, 
and it assumes there is no interaction among attributes, which is not universal in many practical problems. In fact, attribute interactions are unavoidable in certain cases. For example, considering a smart vehicle service system, customers require one product attribute of "high dynamic performance of engine", which interacts with and weakens another product attribute of "low fuel consumption". At the same time, the service attribute "driver behavior monitoring, prediction and recommendation" interacts with and enhances "low fuel consumption". Compared with the additive form, MTPFs have attractive features of describing different attributes interactions simultaneously with only requiring independent targets. However, applications of MTPFs are seldom considered, mainly resulting from the complexity of identifying the coefficients of MTPFs when the number of attributes becomes large. These two identified limitations are the issues to be addressed in the following sections.

The objective of this paper is to develop an approach to process hybrid-information targets and performance levels as well as identify the coefficients of MTPFs to support multi-attribute TBDA. The contributions of this research are twofold. On one hand, a decision maker can express their assessment of performance levels and targets in different forms, and interval numbers, fuzzy numbers, simple linguistic terms, and complex linguistic expressions which are then transformed into probability distributions. After the transformation, there are only two types of forms, including crisp numbers and probability distributions, which further result in four types of comparisons between a performance level and a target in terms of expression forms for measuring probability of target achievement. On the other hand, the multilinear model based on fuzzy measures is introduced to derive MTPFs and, their coefficients are evaluated through fuzzy measures. Equivalence between MTPFs and the multilinear model based on fuzzy measures is formally proved, which enables expressing MTPFs in terms of the multilinear model. Under the context of the multilinear model, the Banzhaf indexes are applied to interpret the interactive behavior of aggregation and 2-additive fuzzy measures are applied to balance the complexity and accuracy of identifying fuzzy measures for the multilinear model. This approach provides a novel way of modeling and solving hybrid-information MTPFs.

The rest of this paper is organized as follows. Section 2 introduces the preliminaries, including TBDA, fuzzy measures and the multilinear model. Section 3 proves the equivalence between MTPFs and the multilinear model. Section 4 proposes the approach for multilinear hybrid-information TBDA. Section 5 applies the proposed approach to a case study involving customer competitive evaluation of smart thermometer patches. Comparisons and discussions are given in Sect. 6. Finally, conclusions are presented in Sect. 7.

\section{Preliminaries}

\subsection{Target-based decision analysis}

Definition 1 (Bordley \& Kirkwood, 2004) A decision maker is defined to be targetoriented for $n$ attributes $X=\left(X_{1}, X_{2}, \ldots, X_{n}\right)$, if the decision maker's utility depends 
only on which targets of $t=\left(t_{1}, t_{2}, \ldots, t_{n}\right)$ are satisfied by the $n$ attributes' performance $x=\left(x_{1}, x_{2}, \ldots, x_{n}\right)$, where there is one target for each attribute.

Let $I=\left(I_{1}, I_{2}, \ldots, I_{n}\right)$ be a set of indicator variables where $I_{i}$ equals to one when $x_{i}$ achieves $t_{i}$ and zero otherwise. Hence, there are $2^{n}$ different possible combinations of target achievement and $2^{n}$ different levels of the utility function $u$ over $I$. Define $I_{x}$ as the set of $2^{n}$ different combinations of target achievement. The expected utility $E(u)$ of a target-based decision maker over $x$ is defined by Bordley and Kirkwood (2004):

$$
E(u)=\int\left[\sum_{I \in I_{x}} u(I) p(I \mid x)\right] f(x) d x
$$

where $u(I)$ is the utility level given target achievement $I, f(x)$ is the probability distribution of $x$, and $p(I \mid x)$ is the probability of target achievement $I$ given $x$.

For notation convenience, define target-based preference function $u_{T}(x)$ by $u_{T}(x)=\sum_{I \in I_{x}} u(I) p(I \mid x)$. Equation (1) shows that target-oriented expected utility $E(u)$ equals the expected value of $u_{T}(x)$.

Definition 2 Targets are said to be independent if a decision maker's probability of achieving the target $t_{i}$ on any attribute $X_{i}$ depends only on the level of $x_{i}$ (Bordley \& Kirkwood, 2004) and not on whether targets for other attributes are achieved or not (Tsetlin \& Winkler, 2007).

With independent target $I_{i}$ 's are probabilistically independent given $x$. In order to present target achievement sets clearly and correspond to fuzzy measures in subsequent work, let $A \subseteq X, A=\left\{i \mid I_{i}=1\right\}$ and $k_{A}=u(I)$. Then, $u_{T}(x)$ becomes a MTPF defined by:

$$
u_{M L}(x)=\sum_{A \subseteq X} k_{A}\left\{\prod_{i \in A} p_{i}\left(x_{i} \succcurlyeq t_{i}\right)\right\}\left\{\prod_{i \notin A}\left[1-p_{i}\left(x_{i} \succcurlyeq t_{i}\right)\right]\right\}
$$

where $p_{i}\left(x_{i} \succcurlyeq t_{i}\right)$ is the probability that target $t_{i}$ is achieved when the performance level is $x_{i}$.

\subsection{Fuzzy measures and multilinear model}

Definition 3 (Beliakov et al., 2020). Let $X=\left\{X_{1}, \ldots, X_{n}\right\}$ be a finite non-empty set of $n$ attributes, and $\mathrm{P}(\mathrm{X})$ be the power set of $X$. A fuzzy measure on $X$ is a set function $\mu: P(X) \rightarrow[0,1]$, satisfying: (1) Boundary conditions: $\mu(\emptyset)=0$ and $\mu(X)=1$, and (2) Monotonicity: if $A \subseteq B \subseteq C$, then $\mu(A) \leq \mu(B) \leq \mu(C)$.

Fuzzy measures are the generalization of weight vectors, taking interactions among attributes into account. For each subset of attributes $S \subseteq P(X), \mu(S)$ can be interpreted as the importance of $S$ and this information is usually used to help guide the aggregation 
of an alternative's individual attribute evaluation to obtain the alternative's overall evaluation to the multiple attributes.

Definition 4 (Beliakov et al., 2020). For any $S \subseteq X$, the Möbius transform of fuzzy measure $\mu(S)$ can be uniquely expressed by:

$$
m_{\mu}(S)=\sum_{A \subseteq S}(-1)^{|S|-|A|} \mu(A)
$$

where $|A|$ and $|S|$ denote cardinalities of subsets $A$ and $S$, respectively. And $m_{\mu}(S)$ satisfies the following conditions: (1) Boundary conditions: $m_{\mu}(\emptyset)=$ 0; $\sum_{S \subseteq X} m_{\mu}(S)=1$, and (2) Monotonicity: $\sum_{\substack{A \subseteq S \\ i \in A}} m_{\mu}(A) \geq 0, \forall S \subseteq X$.

Besides, $\mu(S)$ can be defined in terms of Möbius transform:

$$
\mu(S)=\sum_{A \subseteq S} m_{\mu}(\mathrm{A})
$$

Definition 5 (Beliakov et al., 2020). Let $k \in\{1,2, \ldots, n\}$. A fuzzy measure $\mu$ on $X$ is said to be $k$-additive if its Möbius representation satisfies $m_{\mu}(\mathrm{S})=0$ for all $S \subseteq$ $X,|S|>k$ and there exists at least one subset $S$ of cardinality $k$ such that $m_{\mu}(S) \neq 0$. $k$-additive fuzzy measures can model interactions among at most $k$ attributes.

The multilinear model, an aggregation function, based on fuzzy measures is defined by Grabisch (2016):

$$
F_{M L}(x)=\sum_{S \subseteq X} \mu(S)\left\{\prod_{i \in S} u_{i}\left(x_{i}\right)\right\}\left\{\prod_{i \notin S}\left(1-u_{i}\left(x_{i}\right)\right)\right\}=\sum_{S \subseteq X} m_{\mu}(S) \prod_{i \in S} u_{i}\left(x_{i}\right)
$$

where $u_{i}\left(x_{i}\right)$ represents utility over the attribute $X_{i}$. It is also named as the multilinear extension of pseudo Boolean function (Grabisch, 2016), suitable for modeling interactions among attributes as well as does not need the commensurability assumption.

\section{The connections between multilinear target-based preference functions and multilinear model based on fuzzy measures}

In this section, connections between MTPFs and multilinear model are identified, including the similarity of them in terms of forms, coefficients explanation as well as their strategic equivalence. The equivalence between MTPFs and multilinear model is established under certain conditions, which enables modeling MTPFs using multilinear model.

The proofs need some new notations. Specifically, let $x_{i}^{*}$ and $x^{*}$ be the largest preference level of the attribute $x_{i}$ and the set of attributes $x$, respectively; let $x_{i}^{0}$ and $x^{0}$ be the least preference level of $x_{i}$ and $x$, respectively. Let $\bar{x}_{i}$ denote a performance level of $X_{i}$ 's complement $\bar{X}_{i}$. Hence, $u_{T}(x)$ and $u_{T}\left(x_{i}, \bar{x}_{i}\right)$ can be used interchangeably. Following definition 2 , there is $0 \leq p_{i}\left(x_{i}^{0} \succcurlyeq t_{i}\right) \leq p_{i}\left(x_{i} \succcurlyeq t_{i}\right) \leq p_{i}\left(x_{i}^{*} \succcurlyeq t_{i}\right) \leq$ 
1. Similarly, according to utility theory, there are $u_{i}\left(x_{i}^{0}\right) \leq u_{i}\left(x_{i}\right) \leq u_{i}\left(x_{i}^{*}\right)$ and $u_{T}\left(x^{0}\right) \leq u_{T}(x) \leq u_{T}\left(x^{*}\right)$. Instead of repeating many superscript zeros, we write, for instance, $u_{T}\left(x_{1}, x_{2}, x_{3}^{0}\right)$ as $u_{T}\left(x_{1}, x_{2}\right)$.

To avoid heavy notation, let subscripts represent attributes. For example, let $\{i, j\}$ denote $\left\{X_{i}, X_{j}\right\}$. Furthermore, parentheses or braces of notation or sets will be omitted when it is necessary. For example, let $\mu(i), u_{i}^{\prime}, u_{T}^{*}$ represent $\mu(\{i\}), u_{i}^{\prime}\left(x_{i}\right), u_{T}\left(x^{*}\right)$, respectively.

Lemma 1 There always exists a multilinear function $F_{M L}^{\prime}(x)$ (not necessarily a multilinear model) that is strategically equivalent to a MTPF $u_{M L}(x)$.

Proof According to Bordley and Kirkwood (2004), Eq. (2) can be defined by:

$$
u_{M L}(x)=u_{M L}\left(x_{i}, \bar{x}_{i}\right)=b_{i}\left(\bar{x}_{i}\right)+a_{i}\left(\bar{x}_{i}\right) p_{i}\left(x_{i} \succcurlyeq t_{i}\right)
$$

where $b_{i}\left(\bar{x}_{i}\right)$ and $a_{i}\left(\bar{x}_{i}\right)$ are not arbitrary, because they are implicitly defined by Eq. (2).

Let

$$
\begin{gathered}
F_{M L}^{\prime}(x)=\frac{u_{M L}(x)-u_{M L}^{0}}{u_{M L}^{*}-u_{M L}^{0}} \\
u_{i}^{\prime}\left(x_{i}\right)=\frac{p_{i}\left(x_{i} \succcurlyeq t_{i}\right)-p_{i}\left(x_{i}^{0} \succcurlyeq t_{i}\right)}{p_{i}\left(x_{i}^{*} \succcurlyeq t_{i}\right)-p_{i}\left(x_{i}^{0} \succcurlyeq t_{i}\right)}
\end{gathered}
$$

From Eqs. (7) and (8), it must be true that $F_{M L}^{\prime}(x)$ and $u_{i}^{\prime}\left(x_{i}\right)$ are scaled to lie between zero and one, and that $F_{M L}^{\prime}\left(x^{*}\right)=u_{i}^{\prime}\left(x_{i}^{*}\right)=1, F_{M L}^{\prime}\left(x^{0}\right)=u_{i}^{\prime}\left(x_{i}^{0}\right)=0$. Because $F_{M L}^{\prime}(x)$ is a positive affine transformation of $u_{M L}(x)$, then $F_{M L}^{\prime}(x)$ and $u_{M L}(x)$ are strategically equivalent.

Solve Eqs. (7) and (8) for $u_{M L}(x)$ and $p_{i}\left(x_{i} \succcurlyeq t_{i}\right)$, respectively, and substitute them into Eq. (6). This yields:

$$
\begin{aligned}
F_{M L}^{\prime}(x) & =F_{M L}^{\prime}\left(x_{i}, \overline{x_{i}}\right)=\frac{\bar{b}_{i}+\bar{a}_{i} p_{i}\left(x_{i}^{0} \succcurlyeq t_{i}\right)-u_{M L}^{0}}{u_{M L}^{*}-u_{M L}^{0}}+\frac{\bar{a}_{i}\left[p_{i}\left(x_{i}^{*} \succcurlyeq t_{i}\right)-p_{i}\left(x_{i}^{0} \succcurlyeq t_{i}\right)\right]}{u_{M L}^{*}-u_{M L}^{0}} \\
u_{i}^{\prime} & =\bar{b}_{i}^{\prime}+\bar{a}_{i}^{\prime} u_{i}^{\prime}
\end{aligned}
$$

Substituting $x=\left(x_{i}^{0}, \bar{x}_{i}\right)$ and $x=\left(x_{i}^{*}, \bar{x}_{i}\right)$ into above formula, it yields $\bar{b}_{i}^{\prime}=$ $F_{M L}^{\prime}\left(x_{i}^{0}, \overline{x_{i}}\right)$ and $\bar{b}_{i}^{\prime}+\bar{a}_{i}^{\prime}=F_{M L}^{\prime}\left(x_{i}^{*}, \overline{x_{i}}\right)$. Solving these for $\bar{a}_{i}^{\prime}$ and $\bar{b}_{i}^{\prime}$ and rearranging terms gives:

$$
F_{M L}^{\prime}(x)=u_{i}^{\prime} F_{M L}^{\prime}\left(x_{i}^{*}, \overline{x_{i}}\right)+\left[1-u_{i}^{\prime}\right] F_{M L}^{\prime}\left(x_{i}^{0}, \overline{x_{i}}\right)
$$


Substitute (9) repeatedly into itself for $i=1,2, \ldots, n$ and rearrange terms to get the following result:

$$
\begin{aligned}
F_{M L}^{\prime}(x)= & \sum_{i=1}^{n} m(i) u_{i}^{\prime}+\sum_{i=1}^{n} \sum_{j>i} m(i, j) u_{i}^{\prime} u_{j}^{\prime}+\sum_{i=1}^{n} \sum_{j>i} \sum_{e>j} m(i, j, e) u_{i}^{\prime} u_{j}^{\prime} u_{e}^{\prime} \\
& +\cdots+m(1,2, \ldots, n) u_{1}^{\prime} u_{2}^{\prime} \ldots u_{n}^{\prime}
\end{aligned}
$$

where

$$
\begin{aligned}
& m(i)=F_{M L}^{\prime}\left(x_{i}^{*}, x_{i}^{0}\right)=F_{M L}^{\prime}\left(x_{i}^{*}\right) \\
& m(i, j)=F_{M L}^{\prime}\left(x_{i}^{*}, x_{j}^{*}\right)-F_{M L}^{\prime}\left(x_{i}^{*}\right)-F_{M L}^{\prime}\left(x_{j}^{*}\right) \\
& m(i, j, e)=F_{M L}^{\prime}\left(x_{i}^{*}, x_{j}^{*}, x_{e}^{*}\right)-F_{M L}^{\prime}\left(x_{i}^{*}, x_{j}^{*}\right)-F_{M L}^{\prime}\left(x_{i}^{*}, x_{e}^{*}\right) \\
& \quad-F_{M L}^{\prime}\left(x_{j}^{*}, x_{e}^{*}\right)+F_{M L}^{\prime}\left(x_{i}^{*}\right)+F_{M L}^{\prime}\left(x_{j}^{*}\right)+F_{M L}^{\prime}\left(x_{e}^{*}\right) \\
& m(1,2, \ldots, n)=F_{M L}^{\prime}\left(x_{1}^{*}, x_{2}^{*}, \ldots, x_{n}^{*}\right)-\sum_{i=1}^{n} F_{M L}^{\prime}\left(x_{i}^{*}\right)+\cdots+(-1)^{n-2} \sum_{i=1, j>i}^{n} F_{M L}^{\prime}\left(x_{i}^{*}, x_{j}^{*}\right) \\
& \quad+(-1)^{n-1} \sum_{i=1}^{n} F_{M L}^{\prime}\left(x_{i}^{*}\right)
\end{aligned}
$$

Hence $F_{M L}^{\prime}(x)$ is definitively a multilinear function.

Proposition $1 \mathrm{~A}$ MTPF $u_{M L}(x)$ is strategically equivalent to a multilinear model $F_{M L}(x)$.

Proof Let $m^{\prime}(A)$ denote constants $m(i), m(i, j), m(i, j, e), \ldots, m(1,2, \ldots, n)$, where $A \subseteq X$. For example, if $A=\left\{X_{1}, X_{2}\right\}$, then $m^{\prime}(A)=m\left(X_{1}, X_{2}\right)=m(1,2)$. Let $\mu^{\prime}(A)$ denote the value of $F_{M L}^{\prime}$ when $\left\{x_{i} \mid i \in A\right\}$ are of the largest preference levels and $\left\{x_{i} \mid i \in \bar{A}\right\}$ are of the least preference levels. For example, if $A=\left\{X_{1}, X_{2}\right\}$, then $\mu^{\prime}(A)=\mu^{\prime}(1,2)=F_{M L}^{\prime}\left(x_{1}^{*}, x_{2}^{*}\right)$.

Therefore, Eq. (10) can be simplified to:

$$
F_{M L}^{\prime}(x)=\sum_{A \subseteq X} m^{\prime}(A) \prod_{i \in A} u_{i}^{\prime}
$$

where $m^{\prime}(A)=\sum_{S \subseteq A}(-1)^{|A|-|S|} \mu^{\prime}(S)$.

Obviously, $\mu^{\prime}(\bar{A})$ is a fuzzy measure and $m^{\prime}(A)$ is the corresponding Möbius transform of the fuzzy measure. Therefore, $F_{M L}^{\prime}(x)$ is the multilinear model based on fuzzy measures. Based on Lemma $1, u_{M L}(x)$ is then strategically equivalent to multilinear model. To avoid notation confusion, the multilinear model, fuzzy measures and their Möbius transforms are designated by $F_{M L}(x), \mu(A)$ and $m_{\mu}(A)$, respectively. 
Proposition 2 A MTPF $u_{M L}(x)$ and a multilinear model $F_{M L}(x)$ are equivalent when $u_{T}\left(x^{*}\right)=p_{i}\left(x_{i}^{*} \succcurlyeq t_{i}\right)=1$ and $u_{T}\left(x^{0}\right)=p_{i}\left(x_{i}^{0} \succcurlyeq t_{i}\right)=0$.

Proof Similar to the multi-attribute utility theory, a MTPF can be scaled so that $u_{T}\left(x^{*}\right)=1$ and $u_{T}\left(x^{0}\right)=0$ for some $x^{*}$ and $x^{0}$. And the probability of target achievement $p_{i}\left(x_{i} \succcurlyeq t_{i}\right)$ can be scaled so that $p_{i}\left(x_{i}^{*} \succcurlyeq t_{i}\right)=1$ and $p_{i}\left(x_{i}^{0} \succcurlyeq t_{i}\right)=0$. If these conditions are satisfied, then $F_{M L}(x)=u_{M L}(x)$ and $u_{i}\left(x_{i}\right)=p_{i}\left(x_{i} \succcurlyeq t_{i}\right)$ according to Eqs. (7) and (8), which shows the equivalence between a multilinear model and a MTPF.

Remark $1 u(I)=k_{A}=\mu(A)$ should lie between zero and one, which is a necessary condition for equivalence.

Furthermore, the interpretation of coefficients between a multilinear model and a MTPF is consistent. In multilinear model, $\mu(A)$ is the global evaluation of the alternative that fully satisfies attributes in the subset $A$ and fails to satisfy the remaining ones (Beliakov et al., 2020). In the MTPF, $k_{A}$ is the utility of the alternative where attribute targets in $A$ are completely achieved and other attributes targets entirely fail to be achieved. Comparing these two types of coefficients, both $\mu(A)$ and $k_{A}$ can be interpreted as the overall assessment of the binary alternative $\left(1_{A}, 0_{\bar{A}}\right)$, representing the weight or importance of subset $A$.

On account of complete equivalence, the multilinear model based on fuzzy measures provides a feasible and alternative solution for modeling and solving MTPFs.

\section{An integrated approach for multilinear hybrid-information target-based decision analysis}

Following proposition 2 and remark 1 , a MTPF $u_{M L}(x)$ can be expressed by:

$$
\begin{aligned}
u_{M L}(x) & =\sum_{S \subseteq X} \mu(S)\left\{\prod_{i \in S} p_{i}\left(x_{i} \succcurlyeq t_{i}\right)\right\}\left\{\prod_{i \notin S}\left[1-p_{i}\left(x_{i} \succcurlyeq t_{i}\right)\right]\right\} \\
& =\sum_{S \subseteq X} m_{\mu}(S) \prod_{i \in S} p_{i}\left(x_{i} \succcurlyeq t_{i}\right)
\end{aligned}
$$

where $\mu(S)$ 's are fuzzy measures and $m_{\mu}(S)$ 's are Möbius transforms.

Let $f_{i}\left(x_{i}\right)$ be the probability distribution of $x_{i}$. Assuming $f_{i}\left(x_{i}\right)$ 's are mutually probability independent, then the multilinear target-based expected utility function $E\left(u_{M L}\right)$ is defined by:

$$
E\left(u_{M L}\right)=\int_{R^{n}} u_{M L}(x) f(x) d x=\sum_{S \subseteq X} m_{\mu}(S) \prod_{i \in S} \int p_{i}\left(x_{i} \succcurlyeq t_{i}\right) f_{i}\left(x_{i}\right) d x_{i}
$$

It is necessary to calculate all $\int p_{i}\left(x_{i} \succcurlyeq t_{i}\right) f_{i}\left(x_{i}\right) d x_{i}$ in Eq. (13). Let $E\left(p_{i}\left(x_{i} \succcurlyeq t_{i}\right)\right)=$ $\int p_{i}\left(x_{i} \succcurlyeq t_{i}\right) f_{i}\left(x_{i}\right) d x_{i}$ and it denotes the probability of target achievement of $x_{i} . t_{i}$ and 
$x_{i}$ can be expressed by hybrid information with different forms. These different forms of expressions except crisp numbers are all transformed into probability distributions for calculating the probability of target achievement.

At the same time, Eq. (13) is completely specified by $2^{n}$ number of $m_{\mu}(S)$ or $\mu(S)$. However, it is difficult to identify these coefficients when the number of attributes is large. 2-additive fuzzy measures are a specific case of $k$-additive fuzzy measures. They allow to model interactions between any two attributes and the number of coefficients needed to be determined is $\left(\begin{array}{l}n \\ 2\end{array}\right)$. Therefore, 2-additive fuzzy measures enable to find a trade-off between complexity of identifying fuzzy measures and accuracy of modeling interactions, which are introduced to solve the coefficients in $E\left(u_{M L}\right)$.

\subsection{Measuring probability of target achievement considering hybrid information}

\subsubsection{Different forms of performance levels and targets and their transformations into probability distributions}

Due to the inherent complexity of problems and different levels of cognition and knowledge of decision makers, $x_{i}$ 's and $t_{i}$ 's are usually of different forms, such as crisp numbers, interval numbers, fuzzy numbers, probability distributions, and linguistic terms. If the problems can be clearly defined and decision makers have a deep understanding and knowledge about them, then $x_{i}$ 's and $t_{i}$ 's tend to be assessed more precisely, which may have the form of crisp values or probability distributions. However, if the problems are complex, ill-defined, or unstructured, then decision makers tend to have subjective, fuzzy and linguistic assessments to describe the vague and imprecise information (Lin et al., 2021). Hence $x_{i}$ 's and $t_{i}$ 's are more likely to be expressed by fuzzy numbers or linguistic terms. For example, about the aesthetics of a developing smart wearable device, a decision maker would like to use low, high, or very high to express $x_{i}$ and $t_{i}$. The decision makers sometimes even use more complex linguistic expressions, such as hesitant fuzzy linguistic term sets (HFLTSs) (Lin et al., 2020; Lin et al., 2021; Wang et al., 2017) and linguistic terms with weakened hedges (LTWHs) (Wang et al., 2018). For example, a decision maker may use such HFLTSs as at least high, lower than high and greater than high.

Except crisp numbers, these different forms of $x_{i}$ 's and $t_{i}$ 's are transformed into probability distributions to enable measuring the probability of target achievement. Different forms of $x_{i}$ 's and $t_{i}$ 's and their transformation are given in Fig. 1.

Let $p_{x_{i}}\left(x_{i}^{\#}\right)$ be the probability distribution of $x_{i}$ and $x_{i}^{\#}$ be a specific level belonging to $x_{i}$. Let $p_{t_{i}}\left(t_{i}^{\#}\right)$ be probability distribution of $t_{i}$ and $t_{i}^{\#}$ be a specific level belonging to $t_{i}$. As the transformation from different forms into probability distributions is the same for $x_{i}$ and $t_{i}$, the transformation for $t_{i}$ is presented.

When $t_{i}$ is an interval number, it represents that $t_{i}$ is difficult to be defined and that it falls into an interval, which can be expressed by $t_{i}=\left[t_{i}^{\min }, t_{i}^{\max }\right], t_{i}^{\min }<t_{i}^{\max }$. $p_{t_{i}}\left(t_{i}^{\#}\right)$ is able to be expressed by different probability distributions, such as uniform, normal, lognormal and beta distributions. It depends on decision makers to assess a reasonable probability distribution for $t_{i}$. If there is an equal probability of occurrence 


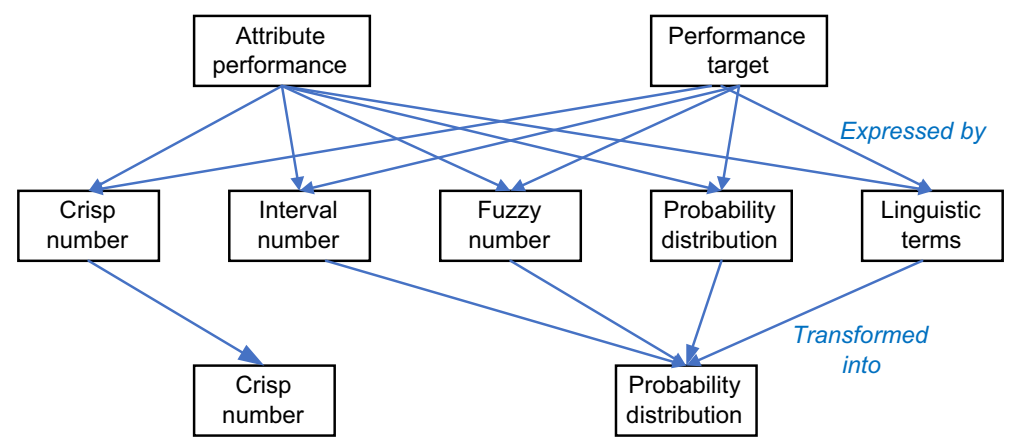

Fig. 1 Different forms of performance levels and targets and their transformation

of $t_{i}^{\#}$ in $t_{i}, p_{t_{i}}\left(t_{i}^{\#}\right)$ is a uniform distribution and it is defined by:

$$
p_{t_{i}}\left(t_{i}^{\#}\right)= \begin{cases}\frac{1}{t_{i}^{\max }-t_{i}^{\min }}, & t_{i}^{\min } \leq t_{i}^{\#} \leq t_{i}^{\max } \\ 0, & \text { otherwise }\end{cases}
$$

When $t_{i}$ is a fuzzy number, it represents that there is fuzziness or imprecision of human cognitive process of $t_{i}$. $t_{i}^{\#}$ has a certain degree of memberships, and its membership function is modeled by:

$$
\mu_{t_{i}}\left(t_{i}^{\#}\right)= \begin{cases}h_{t_{i}}\left(t_{i}^{\#}\right), & t_{i}^{\text {min }} \leq t_{i}^{\#} \leq t_{i}^{l} \\ 1, & t_{i}^{l} \leq t_{i}^{\#} \leq t_{i}^{r} \\ g_{t_{i}}\left(t_{i}^{\#}\right), & t_{i}^{r} \leq t_{i}^{\#} \leq t_{i}^{\text {max }} \\ 0, & \text { otherwise }\end{cases}
$$

where $0 \leq \mu_{t_{i}}\left(t_{i}^{\#}\right) \leq 1, h_{t_{i}}\left(t_{i}^{\#}\right)$ is a real-valued function that is monotonically increasing, and $g_{t_{i}}\left(t_{i}^{\#}\right)$ is a real-valued function that is monotonically decreasing. If $h_{t_{i}}\left(t_{i}^{\#}\right)$ and $g_{t_{i}}\left(t_{i}^{\#}\right)$ are linear functions, then $\mu_{t_{i}}\left(t_{i}^{\#}\right)$ is called a trapezoidal fuzzy number and denoted by $\left(t_{i}^{\text {min }}, t_{i}^{l}, t_{i}^{r}, t_{i}^{\text {max }}\right)$. Furthermore, if $t_{i}^{l}=t_{i}^{r}$, then $\mu_{t_{i}}\left(t_{i}^{\#}\right)$ is called a triangular fuzzy number (TriFN) and denoted by $\left(t_{i}^{\min }, t_{i}^{l}, t_{i}^{\max }\right)$. As there are multiple types of $\mu_{t_{i}}\left(t_{i}^{\#}\right)$, different probability distributions can be obtained.

The issue of transforming a membership function into a probability distribution has been discussed extensively. A proportional method is introduced (Yan et al., 2013), which is defined by:

$$
p_{t_{i}}\left(t_{i}^{\#}\right)=\frac{\mu_{t_{i}}\left(t_{i}^{\#}\right)}{\int_{t_{i}^{\min }}^{t^{\max }} \mu_{t_{i}}\left(t_{i}^{\#}\right) d t_{i}^{\#}}
$$

When $t_{i}$ is a linguistic term, $t_{i}$ takes the form of words and expressions that are constructed in natural or artificial languages. A set of linguistic terms are collected by a linguistic term set. A set of $E+1$ linguistic terms can be denoted by:

$$
L=\left\{l_{p} \mid p=0,1, \ldots, E\right\}, t_{j} \in L
$$


where $E$ is usually an even number. $l_{p}$ is a linguistic term, and its semantics is a fuzzy number. For convenience, $l_{p}$ is usually represented by a TriFN defined in the domain $[0,1]$. Thus, the semantics of a linguistic term $t_{i}$ can be defined by:

$$
\mu_{t_{i}}\left(t_{i}^{\#}\right)= \begin{cases}\left(0,0, \frac{1}{E}\right), & p=0 \\ \left(\frac{p-1}{E}, \frac{p}{E}, \frac{p+1}{E}\right), & p=1, \ldots, E-1 \\ \left(\frac{E-1}{E}, 1,1\right), & P=E\end{cases}
$$

The target $t_{i}$ can also be expressed by complex linguistic terms using the linguistic terms in the linguistic term set. A HFLTS includes several linguistic terms together in a comparative linguistic expression (Wang et al., 2017). The comparative linguistic expression can be lower than $l_{p}$, greater than $l_{p}$, at least $l_{p}$, at most $l_{p}$, between $l_{p}$ and $l_{q}, 0 \leq p<q \leq E$. For example, $L=\left\{l_{0}=\right.$ nothing, $l_{1}=$ defenitely low, $l_{2}=$ very low, $l_{3}=$ low, $l_{4}=$ medium, $l_{5}=$ high, $l_{6}=$ very high, $l_{7}=$ defenitely high, $l_{8}=$ perfect $\}$, the expression "at least high" describes the subset $\left\{l_{5}, l_{6}, l_{7}, l_{8}\right\}$ and "at most high" describes the subset $\left\{l_{0}, l_{1}, l_{2}, l_{3}, l_{4}, l_{5}\right\}$. An uncertain linguistic term (ULT) that expresses a linguistic argument locating between two different terms is covered by a HFLTS. For convenience, researchers have proposed to use the linguistic interval $\left[l_{p}, l_{q}\right]$ instead of $\left\{l_{p}, l_{p+1}, \ldots, l_{q}\right\}$. When $t_{i}$ is a HFLTS $\left[l_{p}, l_{q}\right]$, the semantics of $\left[l_{p}, l_{q}\right]$ is defined based on envelop in the domain $[0,1]$, which is a TraFN:

$$
\mu_{t_{i}}\left(t_{i}^{\#}\right)= \begin{cases}\left(0,0, \frac{q}{E}, \frac{q+1}{E}\right), & p=0, q \neq E \\ \left(\frac{p-1}{E}, \frac{p}{E}, \frac{q}{E}, \frac{q+1}{E}\right), & p \neq 0, q \neq E \\ \left(\frac{p-1}{E}, \frac{p}{E}, 1,1\right), & p \neq 0, q=E\end{cases}
$$

Being different from HFLTSs that include several linguistic terms together, the purpose of LTWHs is to model the uncertainty of using a single linguistic term, which is also frequently used in natural language. The compositions of the LTWHs are weakened hedge and atomic term (Wang et al., 2018). The frequently used set of weakened hedges with increasing weakened power in the sequel is denoted by:

$$
H=\left\{h_{0} \text { :definitely, } h_{1} \text { :more or less, } h_{2} \text { :roughly }\right\}
$$

A LTWH is thus expressed by $\left\langle h_{k}, l_{p}\right\rangle, k=0,1,2$. For example, the LTWH can be definitely high, more or less high, and roughly high. When $t_{i}$ is a LTWH expressed by $\left\langle h_{k}, l_{p}\right\rangle$, the semantics of $t_{i}$ can be simply expressed by a TriFN defined in the domain $[0,1]$ :

$$
\mu_{t_{i}}\left(t_{i}^{\#}\right)=\left(\frac{p-k-1}{E}, \frac{p}{E}, \frac{p+k+1}{E}\right)
$$

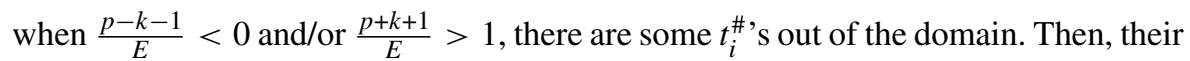
membership degrees are zero. 
Eqs. $(18,19)$ and Eq. (21) then can be transformed straightforwardly into probability distributions using Eq. (16).

\subsubsection{Probability of target achievement under three types of target preferences}

There are three types of target preferences, including benefit form, cost form and interval form (Yan et al., 2013). For these three types of target preferences, the procedures for measuring probability of target achievement are different. Furthermore, after transforming interval numbers, fuzzy numbers and linguistic terms into probability distributions, there are only two different forms of expression for $x_{i}$ and $t_{i}$, namely, crisp numbers and probability distributions. Thus, the transformations result in four possible types of comparisons between $x_{i}$ and $t_{i}$, which are presented in Fig. 2.

(1) Probability of target achievement under a benefit form of target preference

when a decision maker has a benefit form of preference towards $X_{i}$ in the domain $\left[x_{i}^{\text {min }}, x_{i}^{\text {max }}\right], t_{i}$ is adjustable and the more the better. Then, there is $p_{i}\left(x_{i} \succcurlyeq t_{i}\right)=$ $p_{i}\left(x_{i} \geq t_{i}\right)$. When both $x_{i}$ and $t_{i}$ are crisp numbers, $E\left(p_{i}\left(x_{i} \succcurlyeq t_{i}\right)\right)$ is defined by:

$$
E\left(p_{i}\left(x_{i} \succcurlyeq t_{i}\right)\right)=\left\{\begin{array}{l}
1, x_{i} \geq t_{i} \\
0, x_{i}<t_{i}
\end{array}\right.
$$

when $x_{i}$ is a crisp number and $t_{i}$ is expressed by a probability distribution $p_{t_{i}}\left(t_{i}^{\#}\right)$, $E\left(p_{i}\left(x_{i} \succcurlyeq t_{i}\right)\right)$ is defined by:

$$
E\left(p_{i}\left(x_{i} \succcurlyeq t_{i}\right)\right)=\int_{t_{i}^{\text {min }}}^{x_{i}} p_{t_{i}}\left(t_{i}^{\#}\right) d t_{i}^{\#}
$$

\begin{tabular}{|c|c|}
\hline $\begin{array}{c}\text { Type A } \\
\text { performance: crisp number } \\
\text { target: crisp number }\end{array}$ & $\begin{array}{r}\text { Type B } \\
\text { performance: crisp number } \\
\text { target: probability distribution }\end{array}$ \\
\hline $\begin{array}{c}\text { Type C } \\
\text { performance: probability distribution } \\
\text { target: } \text { crisp number }\end{array}$ & $\begin{array}{r}\text { Type D } \\
\text { performance: probability distribution } \\
\text { target: probability distribution }\end{array}$ \\
\hline
\end{tabular}

Fig. 2 Four types of comparisons between a performance level and a target 
when $x_{i}$ is expressed by a probability distribution $p_{x_{i}}\left(x_{i}^{\#}\right)$ and $t_{i}$ is a crisp number, $E\left(p_{i}\left(x_{i} \succcurlyeq t_{i}\right)\right)$ is defined by:

$$
E\left(p_{i}\left(x_{i} \succcurlyeq t_{i}\right)\right)=\int_{t_{i}}^{x_{i}^{\max }} p_{x_{i}}\left(x_{i}^{\#}\right) d x_{i}^{\#}
$$

when $x_{i}$ is expressed by a probability distribution $p_{x_{i}}\left(x_{i}^{\#}\right)$ and $t_{i}$ is expressed by a probability distribution $p_{t_{i}}\left(t_{i}^{\#}\right)$, there are 6 possible cases of position relationships between $\left[x_{i}^{\text {min }}, x_{i}^{\text {max }}\right]$ and $\left[t_{i}^{\text {min }}, t_{i}^{\text {max }}\right]$, including $t_{i}^{\text {min }}<t_{i}^{\text {max }}<x_{i}^{\text {min }}<x_{i}^{\text {max }}$, $x_{i}^{\text {min }}<t_{i}^{\text {min }}<x_{i}^{\text {max }}<t_{i}^{\text {max }}, x_{i}^{\text {min }}<x_{i}^{\text {max }}<t_{i}^{\text {min }}<t_{i}^{\text {max }}$ and so on. For these 6 possible cases, $E\left(p_{i}\left(x_{i} \succcurlyeq t_{i}\right)\right)$ is defined by:

$$
E\left(p_{i}\left(x_{i} \succcurlyeq t_{i}\right)\right)=\int_{x_{i}^{\text {min }}}^{x_{i}^{\text {max }}} \int_{t_{i}^{\text {min }}}^{x_{i}^{\#}} p_{x_{i}}\left(x_{i}^{\#}\right) p_{t_{i}}\left(t_{i}^{\#}\right) d t_{i}^{\#} d x_{i}^{\#}
$$

(2) Probability of target achievement under a cost form of target preference

when a decision maker has a cost form of preference towards $X_{i}$ in the domain $\left[x_{i}^{\min }, x_{i}^{\max }\right], t_{i}$ is adjustable and the less the better. Then, there is $p_{i}\left(x_{i} \succcurlyeq t_{i}\right)=$ $p_{i}\left(x_{i} \leq t_{i}\right)$. When both $x_{i}$ and $t_{i}$ are crisp numbers, $E\left(p_{i}\left(x_{i} \succcurlyeq t_{i}\right)\right)$ is defined by:

$$
E\left(p_{i}\left(x_{i} \succcurlyeq t_{i}\right)\right)=\left\{\begin{array}{l}
1, x_{i} \leq t_{i} \\
0, x_{i}>t_{i}
\end{array}\right.
$$

when $x_{i}$ is a crisp number and $t_{i}$ is expressed by a probability distribution $p_{t_{i}}\left(t_{i}^{\#}\right)$, $E\left(p_{i}\left(x_{i} \succcurlyeq t_{i}\right)\right)$ is defined by:

$$
E\left(p_{i}\left(x_{i} \succcurlyeq t_{i}\right)\right)=\int_{x_{i}}^{t_{i}^{\max }} p_{t_{i}}\left(t_{i}^{\#}\right) d t_{i}^{\#}
$$

when $x_{i}$ is expressed by a probability distribution $p_{x_{i}}\left(x_{i}^{\#}\right)$ and $t_{i}$ is a crisp number, $E\left(p_{i}\left(x_{i} \succcurlyeq t_{i}\right)\right)$ is defined by:

$$
E\left(p_{i}\left(x_{i} \succcurlyeq t_{i}\right)\right)=\int_{x_{i}^{\text {min }}}^{t_{i}} p_{x_{i}}\left(x_{i}^{\#}\right) d x_{i}^{\#}
$$

when $x_{i}$ is expressed by a probability distribution $p_{x_{i}}\left(x_{i}^{\#}\right)$ and $t_{i}$ is expressed by a probability distribution $p_{t_{i}}\left(t_{i}^{\#}\right)$, there are also 6 possible cases of position relationships 
between $\left[x_{i}^{\text {min }}, x_{i}^{\max }\right]$ and $\left[t_{i}^{\min }, t_{i}^{\max }\right]$. For these 6 possible cases, $E\left(p_{i}\left(x_{i} \succcurlyeq t_{i}\right)\right)$ is defined by:

$$
E\left(p_{i}\left(x_{i} \succcurlyeq t_{i}\right)\right)=\int_{x_{i}^{\text {min }}}^{x_{i}^{\text {max }}} \int_{x_{i}^{\#}}^{t_{i}^{\text {max }}} p_{x_{i}}\left(x_{i}^{\#}\right) p_{t_{i}}\left(t_{i}^{\#}\right) d t_{i}^{\#} d x_{i}^{\#}
$$

(3) Probability of target achievement under an interval form of target preference

when a decision maker has a non-monotonic preference towards $X_{i}$ in the domain $\left[x_{i}^{\text {min }}, x_{i}^{\text {max }}\right], t_{i}$ is fairly fixed. Then, there is $p_{i}\left(x_{i} \geq t_{i}\right)=p_{i}\left(x_{i} \cong t_{i}\right)$. When both $x_{i}$ and $t_{i}$ are crisp numbers, $E\left(p_{i}\left(x_{i} \geq t_{i}\right)\right)$ is defined by:

$$
E\left(p_{i}\left(x_{i} \geq t_{i}\right)\right)=\left\{\begin{array}{l}
1, x_{i}=t_{i} \\
0, x_{i} \neq t_{i}
\end{array}\right.
$$

when $x_{i}$ is a crisp number and $t_{i}$ is expressed by a probability distribution $p_{t_{i}}\left(t_{i}^{\#}\right)$, $E\left(p_{i}\left(x_{i} \geq t_{i}\right)\right)$ is defined by:

$$
E\left(p_{i}\left(x_{i} \geq t_{i}\right)\right)=\left\{\begin{array}{l}
1, x_{i} \in\left[t_{i}^{\min }, t_{i}^{\max }\right] \\
0, \text { otherwise }
\end{array}\right.
$$

when $x_{i}$ is expressed by a probability distribution $p_{x_{i}}\left(x_{i}^{\#}\right)$ and $t_{i}$ is a crisp number, there is $p_{i}\left(x_{i} \cong t_{i}\right)=p_{x_{i}}\left(t_{i}\right)$. When $x_{i}$ is expressed by a probability distribution $p_{x_{i}}\left(x_{i}^{\#}\right)$ and $t_{i}$ is expressed by a probability distribution $p_{t_{i}}\left(t_{i}^{\#}\right), E\left(p_{i}\left(x_{i} \succcurlyeq t_{i}\right)\right)$. is defined by:

$$
E\left(p_{i}\left(x_{i} \succcurlyeq t_{i}\right)\right)=\int_{t_{i}^{\min }}^{t_{i}^{\max }} p_{x_{i}}\left(x_{i}^{\#}\right) d x_{i}^{\#}
$$

Interestingly, when $x_{i}$ is a crisp number and $t_{i}$ is expressed by a probability distribution $p_{t_{i}}\left(t_{i}^{*}\right)$, the CDF of $t_{i}$ may be expressed by different forms, such as linear, convex, concave and S-shaped CDFs. These different forms of CDFs imply distinct risk attitudes and perceptions of utility. For example, given a monotonically increasing attribute $X_{i}$, four different types of CDFs can be obtained through assessing different probability distributions as shown in Fig. 3. In Fig. 3a, a uniform distribution is assessed for $p_{t_{i}}\left(t_{i}^{\#}\right)$ and every target has the same probability. Its corresponding CDF is an increasingly linear form that is consistent with the risk-neutral utility functions. In Fig. 3b, $p_{t_{i}}\left(t_{i}^{\#}\right)$ is increasingly linear and a larger target has a higher probability. Its corresponding CDF is a convex form that is consistent with the risk-prone utility functions. In Fig. $3 \mathrm{c}, p_{t_{i}}\left(t_{i}^{\#}\right)$ is decreasingly linear and a smaller target has a higher probability. Its corresponding CDF is a concave form that is consistent with the risk-averse utility functions. In Fig. 3d, $p_{t_{i}}\left(t_{i}^{\#}\right)$ is increasingly linear in $[0,1]$ and decreasingly linear in $[1,4]$. When $t_{i}=1$, the probability is the largest. Its corresponding CDF is a S-shaped form that is consistent with the S-shaped utility functions. 
(a) linear CDF

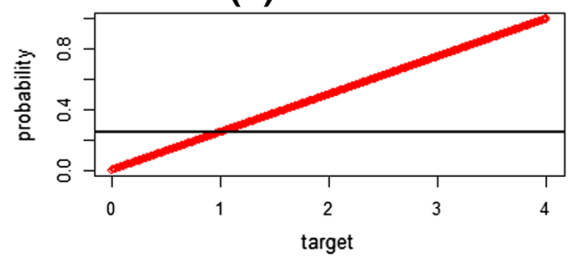

(c) concave CDF

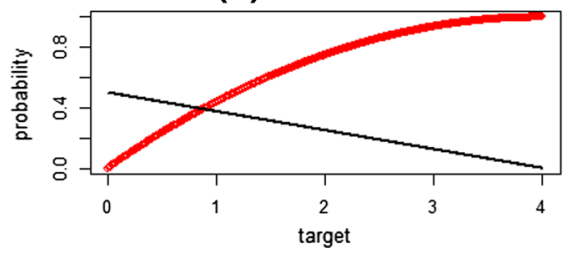

(b) convex CDF

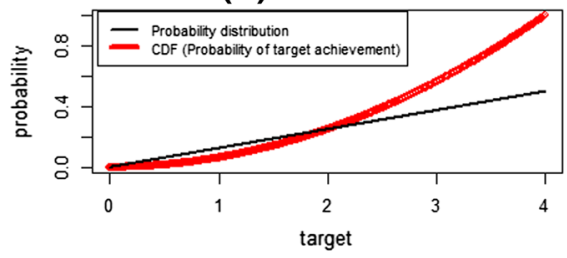

(d) S-shaped CDF

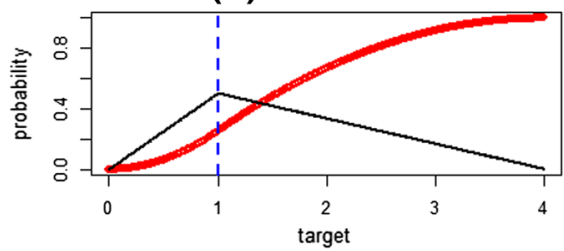

Fig. 3 Four different probability distributions and their corresponding CDFs. Created by Rstudio

\subsection{Expressing multilinear target-oriented preference functions based on multilinear model under 2-additive fuzzy measures}

\subsubsection{Banzhaf index for the multilinear model}

For an intuitive analysis of attributes interaction, interaction index is introduced in the literature. Interaction index describes the interaction degree between elements of a subset $S \subseteq X$, which is the average value of the variation induced by the mixed variation of two attributes or more (Grabisch, 2016).

Definition 4 (Grabisch, 2016). Let $f(x)$ be an integrable aggregation function in $[0,1]^{n}$, and consider $S \neq \emptyset \subseteq X$.

(i) The total variation of $f(x)$ at $x$ with respect to $S$ is the function $\Delta_{S} f(x)$ : $[0,1]^{n} \rightarrow \mathbb{R}$ defined by:

$$
\Delta_{S} f(x)=\Delta_{i}\left(\Delta_{S \backslash i} f\right)(x)
$$

when $S=\{i\}$, then $\Delta_{i} f(x)=f\left(x \mid x_{i}=1\right)-f\left(x \mid x_{i}=0\right)$ is the total variation of $f(x)$ with respect to $i$, which is based on the variation of the aggregated value vs. the total variation of a given attribute, the others being fixed.

(ii) The interaction index of $S$ on $f(x)$ is defined by:

$$
I_{S}(f)=\int_{[0,1]^{n}} \Delta_{S} f(x) d x
$$

Taking $S=\{i\}$, the importance index is obtained, denoting the relative amplitude of the range of $f(x)$ that $i$ may control when assigning random values to the other 
attributes (Grabisch, 2016). The importance index shows the level or percentage of contribution of each attribute in the aggregation process. Obviously, importance index is the special form of interaction index. And $I_{S}(f)$ will be concreted into specific forms when aggregation function $f(x)$ is specified.

Proposition 4 Considering $f(x)$ as the multilinear model $F_{M L}(x)$ in $[0,1]^{n}$, with Möbius representation $m_{\mu}$ of fuzzy measure $\mu$. For any $S \subseteq X$, the interaction index $I_{S}\left(F_{M L}\right)$ is the Banzhaf interaction index $I_{B}(S)$ :

$$
I_{S}\left(F_{M L}\right)=I_{B}(S)=\sum_{T \supseteq S}\left(\frac{1}{2}\right)^{|T|-|S|} m_{\mu}(T)
$$

Proof For any $x \in[0,1]^{n}$, by (5), we have

$$
F_{M L}(x)=\sum_{S \subseteq X} m_{\mu}(S) \prod_{i \in S} x_{i}
$$

which gives

$$
\Delta_{S} F_{M L}(x)=\Delta_{i}\left(\Delta_{S \backslash i} F_{M L}\right)(x)=\sum_{T \supseteq S} m_{\mu}(T) \prod_{i \in T \backslash S} x_{i}
$$

And for any $i \in X$, we get

$$
\int_{[0,1]^{n}} \prod_{i \in X} x_{i} d x=\int_{0}^{1} \int_{0}^{1} \ldots \int_{0}^{1} x_{1} x_{2} \ldots x_{n} d x_{1} d x_{2} \ldots d x_{n}=\frac{1}{2^{n}}
$$

Then

$$
\int_{[0,1]^{n}} \prod_{i \in T \backslash S} x_{i} d x=\frac{1}{2^{|T|-|S|}}
$$

Using (35-1) and (35-2), we finally obtain

$$
\begin{aligned}
I_{S}\left(F_{M L}\right) & =\int_{[0,1]^{n}} \Delta_{S} F_{M L}(x) d x=\int_{[0,1]^{n}} \sum_{T \supseteq S} m_{\mu}(T) \prod_{i \in T \backslash S} x_{i} d x \\
& =\sum_{T \supseteq S}\left[m_{\mu}(T) \int_{[0,1]^{n}} \prod_{i \in T \backslash S} x_{i} d x\right] \\
& =\sum_{T \supseteq S} \frac{1}{2^{|T|-|S|}} m_{\mu}(T)=I_{B}(S)
\end{aligned}
$$

which completes the proof. 
Let $S=\{i\}$, then the Banzhaf importance index $\phi_{B}(i)$ is obtained:

$$
\phi_{B}(i)=I_{B}(i)=\sum_{T \supseteq i}\left(\frac{1}{2}\right)^{|T|-1} m_{\mu}(T)
$$

Similarly, considering $f(x)$ as the Choquet integral $C_{\mu}(x)$ in $[0,1]^{n}, I_{S}\left(C_{\mu}\right)$ is the Shapley interaction index represented by:

$$
I_{s h}(S)=\sum_{T \supseteq S} \frac{1}{|T|-|S|+1} m_{\mu}(T)
$$

And its corresponding Shaley importance index is determined by:

$$
\phi_{S h}(i)=\sum_{S \subseteq X \backslash i} \frac{1}{|S|+1} m_{\mu}(S \cup i)
$$

By the derivation of Banzhaf and Shapley indices, it is natural to use the Banzhaf index in the context of multilinear models, while the Shapley index is more suitable for the Choquet integral.

We can also analyze the adaptability of aggregation functions and interaction indices from the perspective of pseudo-Boolean function. Actually, Eq. (33) in essence is the $s$-derivative of an aggregation function over $[0,1]^{n}$ with respect to $\left\{x_{i}|i \in S| S \mid,=s\right\}$, which is the extension of $s$-derivative of a pseudo-Boolean function $g(x)$ in $\{0,1\}^{n}$ that designated by $\Delta_{S} g(x)$. Just as the multilinear model and the Choquet integrals are multilinear and Lovász extension of pseudo-Boolean function respectively, $\Delta_{S} f(x)$ is regarded as the multilinear extension of $\Delta_{S} g(x)$ when $f(x)$ is the multilinear model while $\Delta_{S} f(x)$ is considered as the Lovász extension of $\Delta_{S} g(x)$ when $f(x)$ is the Choquet integral. In other words, multilinear model and the Banzhaf indices are the application of multilinear extension of pseudo-Boolean function and its $s$-derivative $\Delta_{S} g(x)$ while the Choquet integral and the Shapley index are the application of Lovász extension of them. The relationships among pseudo-Boolean function, its extensions and corresponding interaction index are shown in Fig. 4.

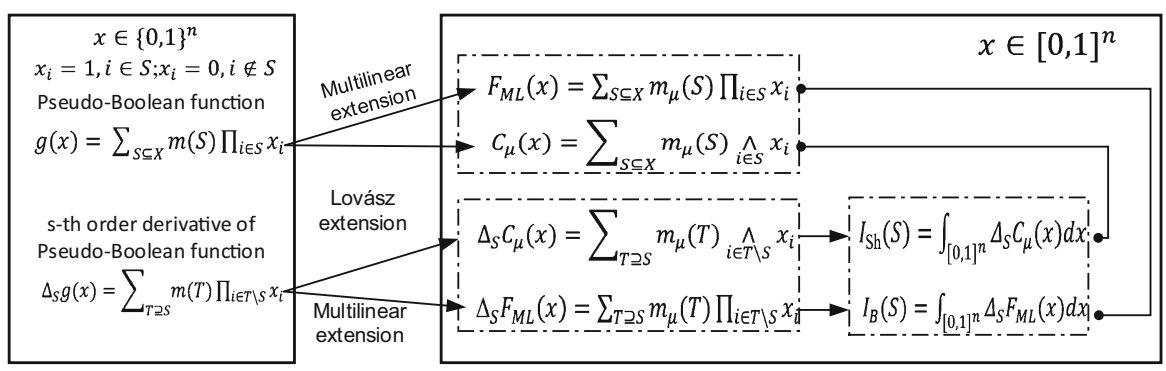

Fig. 4 The relationships among pseudo-Boolean function, its extensions and corresponding interaction index 
$m_{\mu}(S)$ can be rewritten in terms of Banzhaf interaction index by:

$$
m_{\mu}(S)=\sum_{T \supseteq S}\left(-\frac{1}{2}\right)^{|T|-|S|} I_{B}(T)
$$

For 2-additive fuzzy measures, the following equations between $m_{\mu}$ and $I_{B}$ can be derived:

$$
\left\{\begin{array}{l}
\phi_{B}(i)=m_{\mu}(i)+\frac{1}{2} \sum_{\{i, j\} \subseteq X} m_{\mu}(i, j) \\
m_{\mu}(i)=\phi_{B}(i)-\frac{1}{2} \sum_{\{i, j\} \subseteq X} I_{B}(i, j) \\
m_{\mu}(i, j)=I_{B}(i, j) \\
I_{B}(S)=m_{\mu}(S)=0,|S|>2
\end{array}\right.
$$

The 2-additive fuzzy measures only care about interactions between every pair of attributes, that is $I_{B}(i, j)$. An important property of Banzhaf interaction index is that $I_{B}(i, j) \in[-1,1] . I_{B}(i, j)<0$ indicates a negative interaction between $i$ and $j$, which means that it is sufficient to do well in terms of at least one attribute. On the other hand, $I_{B}(i, j)>0$ indicates a positive interaction between $i$ and $j$, which means that it is necessary to satisfy both $i$ and $j$ to perform a good global evaluation. Additionally, $I_{B}(i, j)=0$ indicates that $i$ and $j$ are independent.

\subsubsection{Expressing multilinear target-oriented preference functions based on Banzhaf indices under 2-additive fuzzy measures}

Multilinear target-based expected utility function based on 2-additive fuzzy measures can be defined by the Banzhaf importance index and the Banzhaf interaction index as follows:

$$
\begin{aligned}
& E\left(u_{M L}(x)\right)=\sum_{S \subseteq X} m_{\mu}(S) \prod_{i \in S} E\left(p_{i}\left(x_{i} \succcurlyeq t_{i}\right)\right) \\
& |S| \leq 2 \\
& =\sum_{S \subseteq X} \sum_{T \supseteq S}\left(-\frac{1}{2}\right)^{|T \backslash S|} I_{B}(T) \prod_{i \in S} E\left(p_{i}\left(x_{i} \succcurlyeq t_{i}\right)\right) \\
& |S| \leq 2|T| \leq 2 \\
& =\sum_{S=\emptyset} \sum_{T \supseteq \emptyset}\left(-\frac{1}{2}\right)^{|T|} I_{B}(T)+\sum_{S=\{i\}} \sum_{T \supseteq\{i\}}\left(-\frac{1}{2}\right)^{|T|-1} I_{B}(T) E\left(p_{i}\left(x_{i} \succcurlyeq t_{i}\right)\right) \\
& |T| \leq 2 \quad|T| \leq 2 \\
& +\sum_{S=\{\mathrm{i}, \mathrm{j}\}} \sum_{T \supseteq\{\mathrm{i}, \mathrm{j}\}}\left(-\frac{1}{2}\right)^{|T|-2} I_{B}(T) E\left(p_{i}\left(x_{i} \succcurlyeq t_{i}\right)\right) E\left(p_{j}\left(x_{j} \succcurlyeq t_{j}\right)\right) \\
& |T| \leq 2
\end{aligned}
$$




$$
\begin{aligned}
= & I_{B}(\emptyset)-\frac{1}{2} \sum_{i \in X} I_{B}(i)+\frac{1}{4} \sum_{\{i, j\} \subseteq X} I_{B}(i, j)+\sum_{i \in X} E\left(p_{i}\left(x_{i} \succcurlyeq t_{i}\right)\right)\left(\phi_{B}(i)-\frac{1}{2} \sum_{\{i, j\} \subseteq X} I_{B}(i, j)\right) \\
& +\sum_{\{i, j\} \subseteq X} E\left(p_{i}\left(x_{i} \succcurlyeq t_{i}\right)\right) E\left(p_{j}\left(x_{j} \succcurlyeq t_{j}\right)\right) I_{B}(i, j)
\end{aligned}
$$

Under the 2-additive model, $\mu(\emptyset)$ satisfies:

$$
\mu(\emptyset)=I_{B}(\emptyset)-\frac{1}{2} \sum_{i \in X} I_{B}(i)+\frac{1}{4} \sum_{\{i, j\} \subseteq X} I_{B}(i, j)=0
$$

Substituting $\mu(\emptyset)=0$ into Eq. (41), it yields:

$$
\begin{aligned}
E\left(u_{M L}(x)\right)= & \sum_{i \in X} E\left(p_{i}\left(x_{i} \succcurlyeq t_{i}\right)\right)\left(\phi_{B}(i)-\frac{1}{2} \sum_{\{i, j\} \subseteq X} I_{B}(i, j)\right) \\
& +\sum_{\{i, j\} \subseteq X} E\left(p_{i}\left(x_{i} \succcurlyeq t_{i}\right)\right) E\left(p_{j}\left(x_{j} \succcurlyeq t_{j}\right)\right) I_{B}(i, j)
\end{aligned}
$$

Consequently, Eq. (42) is determined completely by $n \phi_{B}(i)$ and $\frac{n(n-1)}{2} I_{B}(i, j), i, j=1,2, \cdots n$. In particular, under 2-additive fuzzy measures, according to Eqs. (37) and (38), there are $\phi_{S h}(i)=$ $m_{\mu}(i)+\frac{1}{2} \sum_{\{i, j\} \subseteq X} m_{\mu}(i, j)=\phi_{B}(i)$, and $I_{S h}(i, j)=m_{\mu}(i, j)=I_{B}(i, j)$. Therefore, with 2-additive fuzzy measures, Banzhaf importance index and Banzhaf interaction index in multilinear model can be identified through the methods for identifying Shapley importance index and Shapley interaction index in the framework of the Choquet integral.

\subsection{Identification of Banzhaf indices}

Identification of fuzzy measures is an important task in the process of solving $E\left(u_{M L}(x)\right)$. Most of the identification methods have been proposed as optimization problems, such as the least-squares based method, the maximum split method and the minimum variance method (Beliakov et al., 2020). The advantage of the minimum variance method is that it leads to a unique solution because of the strict convexity of objective function. Also, initial preferences on the overall utility of alternatives are not necessary, which might be difficult to elicit. Therefore, the minimum variance method is used to identify 2-additive fuzzy measures. Furthermore, as there is unique correspondence among fuzzy measure, Möbius transform and Banzhaf index, the minimum variance method can be applied to identify Banzhaf indices.

Under 2-additive fuzzy measures, according to definition 4 and Eqs. (36-40), we get:

$$
\mu(X)=\sum_{S \subseteq X} m_{\mu}(S)=\sum_{i \in X} m_{\mu}(i)+\sum_{\{i, j\} \subseteq X} m_{\mu}(i, j)
$$




$$
\begin{aligned}
& =\sum_{i \in X} \phi_{B}(i)-\frac{1}{2} \times 2 \sum_{\{i, j\} \subseteq X} I_{B}(i, j)+\sum_{\{i, j\} \subseteq X} I_{B}(i, j) \\
& =\sum_{i \in X} \phi_{B}(i)=1
\end{aligned}
$$

Because $\phi_{B}(i)$ is the importance index of attribute $X_{i}$ and $\sum_{i \in X} \phi_{B}(i)=1$, we can set:

$$
\phi_{B}(i)=\omega_{i}
$$

where $\omega_{i}$ is the relative importance of $X_{i}$ and can be determined through such weight determination methods as DEMATEL, Analytic Network Process or Analytic Hierarchy Process.

Furthermore, for $\forall S \subseteq X, i \in S$,

$$
\begin{aligned}
\sum_{\{i\} \subseteq A \subseteq S} m_{\mu}(A) & =m_{\mu}(i)+\sum_{j \in S} m_{\mu}(i, j) \\
& =\phi_{B}(i)-\frac{1}{2} \sum_{j \in X \backslash\{i\}} I_{B}(i, j)+\sum_{j \in S \backslash\{i\}} I_{B}(i, j) \\
& =\phi_{B}(i)-\frac{1}{2} \sum_{j \in X \backslash S} I_{B}(i, j)+\frac{1}{2} \sum_{j \in S \backslash\{i\}} I_{B}(i, j) \geq 0
\end{aligned}
$$

The above inequality can be ensured by Wu and Zhang (2010):

$$
\left|I_{B}(i, j)\right| \leq \frac{2}{n-1} \min \left[\phi_{B}(i), \phi_{B}(j)\right]
$$

That is, this inequality is a sufficient condition for $\sum_{\{i\} \subseteq A \subseteq S} m_{\mu}(A) \geq 0$ to be true under 2-additive fuzzy measures. For convenience, let $I_{i j}=\min \left\{\frac{2 \phi_{B}(i)}{n-1}, \frac{2 \phi_{B}(j)}{n-1}\right\}$ and $I_{B}(i, j)$ should be limited into $\left[-I_{i j}, I_{i j}\right]$. For the sake of describing attributes interactions more intuitively, $\left[-I_{i j}, I_{i j}\right]$ is divided into five equal parts, including $\left[-I_{i j},-\frac{3}{5} I_{i j}\right],\left[-\frac{3}{5} I_{i j},-\frac{1}{5} I_{i j}\right],\left[-\frac{1}{5} I_{i j}, \frac{1}{5} I_{i j}\right],\left[\frac{1}{5} I_{i j}, \frac{3}{5} I_{i j}\right],\left[\frac{3}{5} I_{i j}, I_{i j}\right]$, representing significant negative interaction, negative interaction, independence, positive interaction and significant positive interaction, respectively. The interaction degree of any pair of attributes should lie in any subinterval $I_{i j}^{\prime}(i, j=1,2, \ldots, n$ and $i \neq j)$. This yields:

$$
I_{B}(i, j) \in I_{i j}^{\prime}
$$

In summary, the Banzhaf importance indices and interaction indices satisfying Eqs. (43) and (44) will correspond to 2-additive fuzzy measures.

Finally, in spirit of minimum variance principle, the objective function under the constraint conditions of (43) and (44) is constructed as follows: 


$$
\begin{aligned}
& \min z=\frac{1}{n} \sum_{i \in X} \sum_{S \subseteq X \backslash i} \frac{(|X|-|S|-1) !|S| !}{|X| !}\left(\phi_{B}(i)-\frac{1}{2} \sum_{j \in X \backslash S \cup i} I_{B}(i, j)+\frac{1}{2} \sum_{j \in S} I_{B}(i, j)-\frac{1}{n}\right)^{2} \\
& \text { s.t. }\left\{\begin{array}{l}
I_{B}(i, j) \in I_{i j}^{\prime} \\
\phi_{B}(i)=\omega_{i} \\
i, j=1,2, \ldots, n, \text { and } i \neq j \\
\ldots
\end{array}\right.
\end{aligned}
$$

By solving the above nonlinear programming, the Banzhaf interaction indices are identified.

\subsection{The procedures of the proposed approach}

Let $A=\left\{a_{1}, \ldots, a_{j}, \ldots, a_{m}\right\}, j=1, \ldots, m$ be the set of alternatives and $X=$ $\left(X_{1}, \ldots, X_{i}, \ldots, X_{n}\right), i=1, \ldots, n$, be the set of attributes. Let $x_{i j}$ be the performance level of $X_{i}$ given the alternative $a_{j}$. Let $t=\left(t_{1}, \ldots, t_{i}, \ldots, t_{n}\right)$ be the corresponding targets of $X$, where there is a single target $t_{i}$ for $X_{i}$. Assume independent targets among $t_{i}$ 's and probability independence among $x_{i}$ 's can be verified.

Based on Sects. 4.1, 4.2 and 4.3, an integrated approach for multi-attribute hybridinformation TBDA is proposed based on multilinear model and 2-additive fuzzy measures. The approach includes the following steps.

Step 1 Transform diverse forms of $x_{i j}$ and $t_{i}$ into probability distributions $p_{x_{i j}}\left(x_{i j}^{\#}\right)$ and $p_{t_{i}}\left(t_{i}^{\#}\right)$, respectively, using Eqs. (14-21).

Step 2 Determine the specific type of target preferences for the targets $t_{i}$, and measure probability of target achievement $E\left(p_{i j}\left(x_{i j} \succcurlyeq t_{i}\right)\right)$ by considering 4 types of comparing $x_{i j}$ with $t_{i}$ in terms of expression forms, using Eqs. (22-32).

Step 3 Calculate the Banzhaf importance index $\phi_{B}(i)$ and the Banzhaf interaction index $I_{B}(i, j)$. Because of $\phi_{S h}(i)=\phi_{B}(i)$, and $I_{S h}(i, j)=I_{B}(i, j)$ under the case of 2-additive fuzzy measures, $\phi_{B}(i)$ is calculated with DEMATEL while $I_{B}(i, j)$ is calculated from Eq. (45).

Step 4 Substitute the value of $E\left(p_{i j}\left(x_{i j} \succcurlyeq t_{i}\right)\right), \phi_{B}(i)$ and $I_{B}(i, j)$ into Eq. (42) to build the multilinear target-based expected utility function $E\left(u_{M L}(x)\right)$, and determine the ranking of $m$ alternatives.

\section{Illustrative application}

In this section, we conduct a case involving customer competitive evaluation of a set of smart thermometer patches to demonstrate feasibility and validity of proposed approach.

With the rapid development of the new generation of information technologies and the serious challenge posed by the COVID-19 pandemic, wearable and continuously monitoring thermometers with instant fever alerts have broad prospects in hospitals as 
well as in home care settings, which brings opportunities and challenges to the competitive smart healthcare market. Aiming to this emerging market, company A would like to conduct the competitive evaluation of a prototype of a smart thermometer patch in terms of customer needs. In this scenario, the key for company A to keep competitive in the market is determined by how well its product performs relative to the competing ones, which is essentially target-oriented. At the same time, perceived performance levels of existing products already in the market and products under development are usually expressed in different forms, such as crisp numbers, interval numbers, fuzzy numbers, linguistic terms, or complex linguistic expressions. Therefore, it is very natural to conduct customer competitive evaluation of smart thermometer patches from the perspective of multilinear hybrid-information TBDA.

Company A has organized a customer focus group for this customer competitive evaluation and all assessment results are based on group discussions and consensus. By discussion, 8 customer needs and 5 alternatives are identified, as shown in Tables 1 and 2, respectively. Furthermore, taking hybrid-information into account, performance levels of different products and targets are determined in Table 2. The linguistic term set is determined as $L=\left\{l_{0}=\right.$ nothing, $l_{1}=$ defenitely low, $l_{2}=$ very low, $l_{3}=$ low, $l_{4}=$ medium, $l_{5}=$ high, $l_{6}=$ very high, $l_{7}=$ defenitely high, $l_{8}=$ perfect $\}$, used for linguistic assessment.

\subsection{Step 1: transform different forms of performance levels and targets into probability distributions}

As presented in Table 2, the numbers such as 7 days of battery life and 300 Chinese Yuan of purchasing cost are crisp numbers. Also, the binary values "yes" and "no" are special case of crisp numbers. They are kept as they are. For other forms of expressions, they are transformed into probability distributions in order to measure probability of target achievement. All the transformed probability distributions of performance levels and targets are given in Table 3 .

\subsection{Step 2: measure probability of target achievement}

The probability of target achievement $E\left(p_{i}\left(x_{i j} \succcurlyeq t_{i}\right)\right)$ are measured by considering the type of target preferences for $t_{i}$ and the four types of comparison between $x_{i j}$ and $t_{i}$ in terms of expression forms. The type of target preferences for $t_{i}$ are identified and given in Table 2.

For example, there are the following steps to calculate the probability of achieving target $t_{2}$ by product $\mathrm{E} E\left(p_{2}\left(x_{2} E \succcurlyeq t_{2}\right)\right)$. First, the target preference of $t_{2}$ is identified as benefit form. Then, the expression form of the target $t_{2}$ and $x_{2 E}$ are checked and they are both expressed by probability distributions, which results in selecting Eq. (34) to 
Table 1 Definitions of customer needs

\begin{tabular}{|c|c|c|c|}
\hline Customer need & Description & Measure & Monotonicity \\
\hline $\begin{array}{c}X_{1}: \text { Accuracy of } \\
\text { measurement }\end{array}$ & $\begin{array}{l}\text { The deviation from the } \\
\text { result of the mercurial } \\
\text { thermometer }\end{array}$ & $\begin{array}{l}\text { The value of deviation in } \\
\text { terms of degree centigrade }\end{array}$ & $\begin{array}{l}\text { Non } \\
\text { monotonic }\end{array}$ \\
\hline$X_{2}$ : Comfort & $\begin{array}{l}\text { The degree of comfort, } \\
\text { including smooth hand, } \\
\text { thin thickness, good air } \\
\text { permeability and } \\
\text { waterproof }\end{array}$ & $\begin{array}{l}\text { It is assessed by the experts } \\
\text { through their subjective } \\
\text { judgements with linguistic } \\
\text { terms }\end{array}$ & Increasing \\
\hline$X_{3}$ : Ease of use & $\begin{array}{l}\text { The usability of the product } \\
\text { and its App }\end{array}$ & $\begin{array}{l}\text { It is assessed by the experts } \\
\text { through their subjective } \\
\text { judgements with linguistic } \\
\text { terms }\end{array}$ & Increasing \\
\hline$X_{4}:$ Battery life & $\begin{array}{l}\text { The time the product will } \\
\text { run before it must be } \\
\text { recharged }\end{array}$ & $\begin{array}{l}\text { The days can be used before } \\
\text { the product must be } \\
\text { recharged }\end{array}$ & Increasing \\
\hline $\begin{array}{l}X_{5}: \text { Continuous } \\
\text { monitoring }\end{array}$ & $\begin{array}{l}\text { The capability to monitor } \\
\text { the temperature } \\
\text { continuously }\end{array}$ & $\begin{array}{l}\text { If the product can monitor } \\
\text { the temperature } \\
\text { continuously, then the } \\
\text { evaluation is "yes"; } \\
\text { otherwise, "no" }\end{array}$ & Increasing \\
\hline $\begin{array}{l}X_{6}: \text { Remote } \\
\text { monitoring }\end{array}$ & $\begin{array}{l}\text { The capability to monitor } \\
\text { the temperature remotely }\end{array}$ & $\begin{array}{l}\text { If the product can monitor } \\
\text { the temperature remotely, } \\
\text { then the evaluation is } \\
\text { "yes"; otherwise, "no" }\end{array}$ & Increasing \\
\hline $\begin{array}{l}X_{7}: \text { Value-added } \\
\text { service }\end{array}$ & $\begin{array}{l}\text { The extra services, such as } \\
\text { fever alarm and advices } \\
\text { on nursing }\end{array}$ & $\begin{array}{l}\text { It is assessed by the experts } \\
\text { through their subjective } \\
\text { judgements with linguistic } \\
\text { terms }\end{array}$ & Increasing \\
\hline$X_{8}$ : Purchasing cost & $\begin{array}{l}\text { The sale price of the } \\
\text { combined product and } \\
\text { service package }\end{array}$ & Chinese Yuan & Decreasing \\
\hline
\end{tabular}

calculate $E\left(p_{2}\left(x_{2 E} \succcurlyeq t_{2}\right)\right)$.

$$
\begin{aligned}
E\left(p_{2}\left(x_{2 E} \succcurlyeq t_{2}\right)\right) & =E\left(p_{2}\left(x_{2 E} \geq t_{2}\right)\right)=\int_{x_{2 E}^{\min }}^{x_{2 E}^{\max }} \int_{t_{2}^{\min }}^{x_{2 E}^{*}} p_{x_{2 E}}\left(x_{2 E}^{*}\right) p_{t_{2}}\left(t_{2}^{*}\right) d x_{2 E}^{*} d t_{2}^{*} \\
& =\int_{x_{2 E}^{\min }}^{x_{2 E}^{\max }} p_{x_{2 E}}\left(x_{2 E}^{*}\right) \int_{t_{2}^{\min }}^{x_{2 E}^{*}} p_{t_{2}}\left(t_{2}^{*}\right) d t_{2}^{*} d x_{2 E}^{*} \\
& =\int_{0.75}^{0.875} \frac{1}{0.3125}\left(64\left(0.5 x_{2 E}^{* 2}-0.75 x_{2 E}^{*}+0.28125\right)\right) d x_{2 E}^{*}
\end{aligned}
$$


Table 2 Products' performance levels and their corresponding targets

\begin{tabular}{|c|c|c|c|c|c|c|c|}
\hline $\begin{array}{l}\text { Customer } \\
\text { need }\end{array}$ & $\begin{array}{l}\text { target } \\
\text { preference }\end{array}$ & $\begin{array}{l}\text { Product } \\
\text { A }\end{array}$ & $\begin{array}{l}\text { Product } \\
\text { B }\end{array}$ & $\begin{array}{l}\text { Product } \\
\text { C }\end{array}$ & $\begin{array}{l}\text { Product } \\
\text { D }\end{array}$ & $\begin{array}{l}\text { Product } \\
\text { E }\end{array}$ & Target \\
\hline$X_{1}$ & $\begin{array}{l}\text { Interval } \\
\text { form }\end{array}$ & $\begin{array}{c}{[-0.1,} \\
0.1]\end{array}$ & $\begin{array}{c}{[-0.05} \\
0.05]\end{array}$ & $\begin{array}{c}{[-0.1,} \\
0.1]\end{array}$ & $\begin{array}{c}{[-0.02} \\
0.02]\end{array}$ & $\begin{array}{c}{[-0.05} \\
0.05]\end{array}$ & $\begin{array}{c}{[-0.05,} \\
0.05]\end{array}$ \\
\hline$X_{2}$ & $\begin{array}{c}\text { Benefit } \\
\text { form }\end{array}$ & $l_{6}$ & $l_{6}$ & $l_{5}$ & $l_{7}$ & $\begin{array}{l}\text { At least } \\
l_{6}\end{array}$ & $l_{7}$ \\
\hline$X_{3}$ & $\begin{array}{c}\text { Benefit } \\
\text { form }\end{array}$ & $l_{5}$ & $l_{6}$ & $l_{6}$ & $l_{5}$ & $l_{6}$ & $l_{6}$ \\
\hline$X_{4}$ & $\begin{array}{c}\text { Benefit } \\
\text { form }\end{array}$ & 7 & 8 & 5 & 10 & {$[7,10]$} & {$[5,10]$} \\
\hline$X_{5}$ & $\begin{array}{c}\text { Benefit } \\
\text { form }\end{array}$ & Yes & Yes & Yes & Yes & Yes & Yes \\
\hline$X_{6}$ & $\begin{array}{c}\text { Benefit } \\
\text { form }\end{array}$ & Yes & Yes & No & Yes & Yes & Yes \\
\hline$X_{7}$ & $\begin{array}{c}\text { Benefit } \\
\text { form }\end{array}$ & $l_{7}$ & $l_{6}$ & $l_{0}$ & $l_{7}$ & $\begin{array}{c}\text { More or } \\
\text { less } l_{7}\end{array}$ & $l_{7}$ \\
\hline$X_{8}$ & Cost form & 300 & 400 & 200 & 500 & $\begin{array}{l}{[300,} \\
360]\end{array}$ & $\begin{array}{l}{[200,} \\
500]\end{array}$ \\
\hline
\end{tabular}

$$
+\int_{0.875}^{1} \frac{1}{0.3125}\left(-32 x_{2 E}^{* 2}+64 x_{2 E}^{*}-31\right) d x_{2 E}^{*}=0.39999
$$

The calculated probability of target achievement is given in Table 4.

\subsection{Step 3 Calculate the Banzhaf importance index and the Banzhaf interaction index}

$\phi_{B}(i)=\phi_{S h}(i)$ is recognized as overall relative importance of the customer need $X_{i}$ and it is calculated through the DEMATEL method. First, the direct-relation matrix of the 8 customer needs is identified and shown in Table 5. The 5-level scale is used to indicate the influence degrees from one customer need to another customer need (No influence $=0$, Very low influence $=1$, Low influence $=2$, High influence $=3$, Very high influence $=4$ ).

Based on the direct-relation matrix, the algorithm of DEMATEL is applied directly to calculate the prominence, relationship, and relative weight of each customer need. The prominence of $X_{i}$ indicates the total influence degree that is given by and received by $X_{i}$. The relation of $X_{i}$ indicates the net effect that $X_{i}$ contributes to other customer needs. The relative weight of $X_{i}$ is denoted by $w_{i}$ and it is calculated based on the prominence and relation. The results of prominence, relation and relative weights are given in Table 6. 


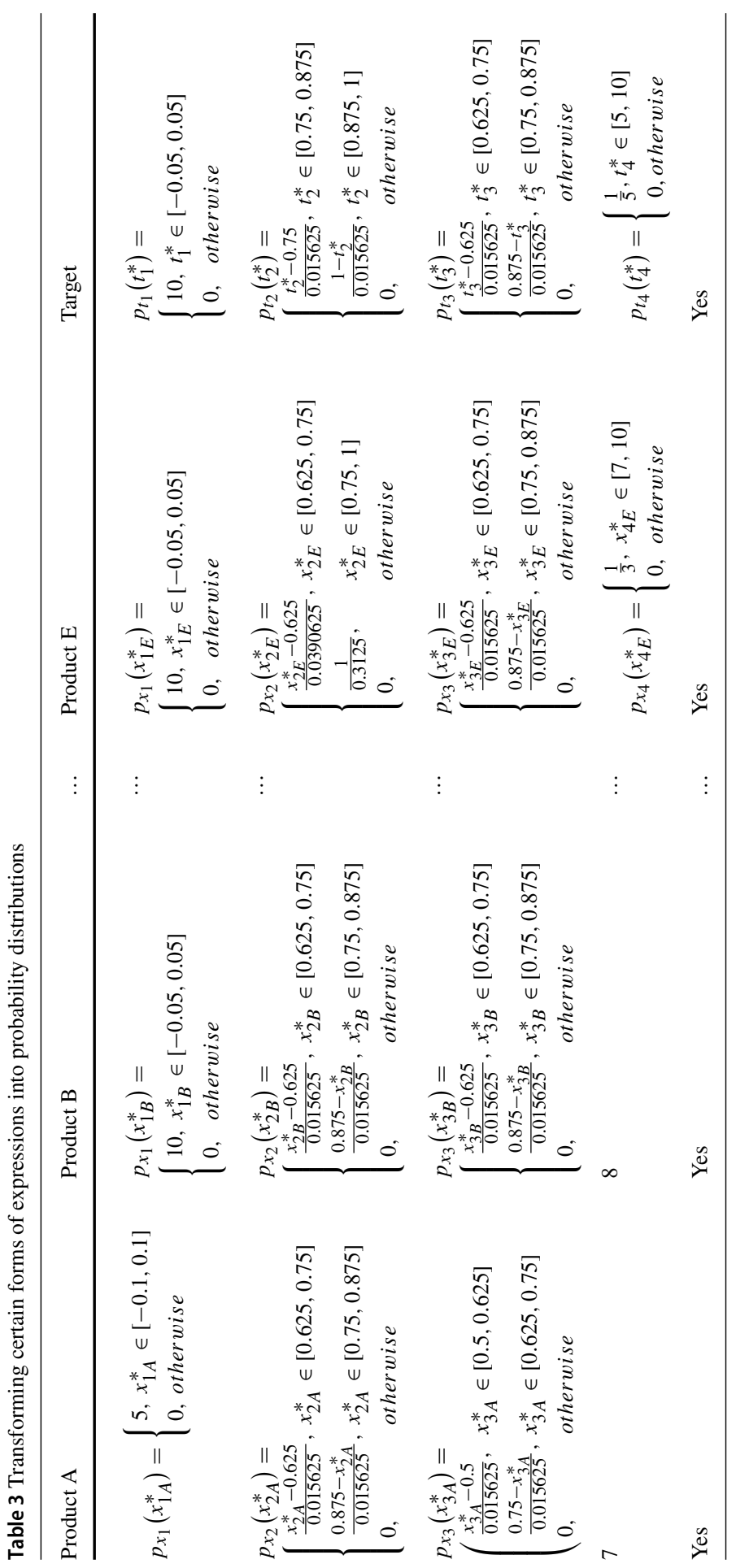




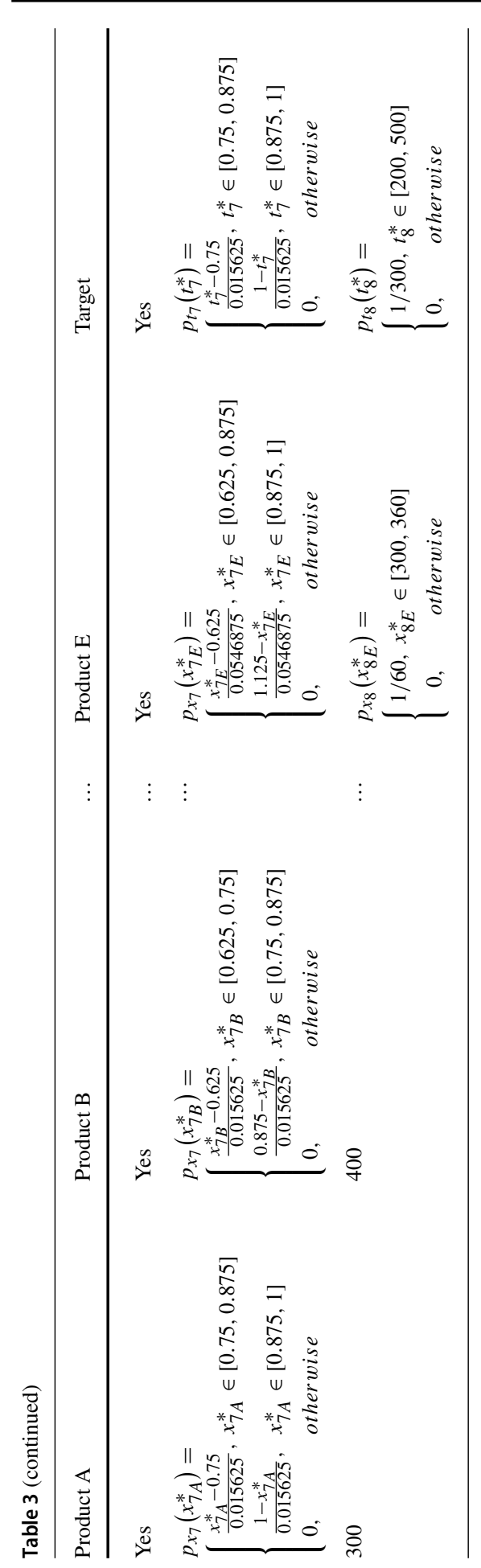


Table 4 Probability of target achievement

\begin{tabular}{llllll}
\hline Customer need & \multicolumn{4}{l}{ Probability of target achievement } & \\
\cline { 2 - 6 } & Product A & Product B & Product C & Product D & Product E \\
\hline$X_{1}$ & 0.5 & 1 & 0.5 & 1 & 1 \\
$X_{2}$ & 0.04166 & 0.04166 & 0 & 0.5 & 0.39999 \\
$X_{3}$ & 0.04166 & 0.5 & 0.5 & 0.04166 & 0.5 \\
$X_{4}$ & 0.4 & 0.6 & 0 & 1 & 0.7 \\
$X_{5}$ & 1 & 1 & 1 & 1 & 1 \\
$X_{6}$ & 1 & 1 & 0 & 1 & 1 \\
$X_{7}$ & 0.5 & 0.04166 & 0 & 0.5 & 0.42857 \\
$X_{8}$ & 0.66667 & 0.33333 & 1 & 0 & 0.56667 \\
\hline
\end{tabular}

Table 5 Direct-relation matrix of customer needs

\begin{tabular}{lllllllll}
\hline Customer need & $X_{1}$ & $X_{2}$ & $X_{3}$ & $X_{4}$ & $X_{5}$ & $X_{6}$ & $X_{7}$ & $X_{8}$ \\
\hline$X_{1}$ & 0 & 0 & 0 & 0 & 0 & 0 & 3 & 1 \\
$X_{2}$ & 2 & 0 & 0 & 0 & 0 & 0 & 0 & 1 \\
$X_{3}$ & 2 & 0 & 0 & 0 & 0 & 0 & 0 & 1 \\
$X_{4}$ & 0 & 0 & 0 & 0 & 3 & 0 & 0 & 1 \\
$X_{5}$ & 0 & 2 & 0 & 0 & 0 & 3 & 0 & 1 \\
$X_{6}$ & 0 & 0 & 3 & 0 & 0 & 0 & 3 & 1 \\
$X_{7}$ & 0 & 0 & 0 & 0 & 0 & 0 & 0 & 2 \\
$X_{8}$ & 0 & 0 & 0 & 0 & 0 & 0 & 0 & 0 \\
\hline
\end{tabular}

According to the conditions to be satisfied under 2-additive fuzzy measures, initial constrains on the degree of interactions are derived according to Eq. (45). Furthermore, the types of interactions with their strength are also identified and collected in Table 7. After combining these two kinds of information, the constrains on degrees of interactions between any pair of requirements are calculated.

With the relative weights of the customer needs and the constrains of degree of interactions, the minimum variance method is applied to identity the fuzzy measures, Möbius transforms, and the Shapley interaction index. The calculated results are given in Table 8.

Then, according to $\phi_{B}(i)=\phi_{S h}(i)$ and $I_{B}(i, j)=I_{S h}(i, j)$, the Banzhaf importance index $\phi_{B}(i)$ and the Banzhaf interaction index $I_{B}(i, j)$ are determined. 
Table 6 Customer needs' relative weights

\begin{tabular}{llrl}
\hline Customer need & Prominence & Relation & Relative weight \\
\hline$X_{1}$ & 1.579 & -0.192 & 0.104 \\
$X_{2}$ & 1.035 & 0.218 & 0.069 \\
$X_{3}$ & 1.318 & -0.064 & 0.086 \\
$X_{4}$ & 1.271 & 1.271 & 0.118 \\
$X_{5}$ & 2.061 & 1.203 & 0.156 \\
$X_{6}$ & 2.003 & 0.779 & 0.141 \\
$X_{7}$ & 1.785 & -1.213 & 0.141 \\
$X_{8}$ & 2.003 & -2.003 & 0.185 \\
\hline
\end{tabular}

\subsection{Step 4 built the multilinear target-based expected utility function and determine the ranking of product alternatives}

For brevity, let $p_{i j}=E\left(p_{i}\left(x_{i j} \succcurlyeq t_{i}\right)\right)$. The multilinear target-based expected utility function based on 2-additive fuzzy measures for evaluating different smart thermometer patch alternatives is then built, and it is defined by:

$$
\begin{aligned}
E\left(u_{M L}(x)\right)= & 0.0935 p_{1 j}+0.054 p_{2 j}+0.0835 p_{3 j} \\
& +0.123 p_{4 j}+0.1685 p_{5 j}+0.1615 p_{6 j} \\
& +0.154 p_{7 j}+0.163 p_{8 j}+0.012 p_{1 j} p_{2 j} \\
& +0.005 p_{1 j} p_{3 j}-0.002 p_{1 j} p_{4 j}+0.001 p_{1 j} p_{5 j} \\
& +0.005 p_{1 j} p_{6 j}-0.006 p_{1 j} p_{7 j}+0.006 p_{1 j} p_{8 j} \\
& +\cdots+0.008 p_{6 j} p_{8 j}+0.008 p_{7 j} p_{8 j}
\end{aligned}
$$

The five product alternatives listed in Table 4 are then evaluated using Eq. (46). The ranking of the five alternatives is the following: $E(0.71293) \succcurlyeq \mathrm{D}(0.60698) \succcurlyeq \mathrm{A}(0.5882) \succcurlyeq \mathrm{B}(0.5802) \succcurlyeq \mathrm{C}(0.43825)$. The results clearly show that the developed prototype A from company $\mathrm{A}$ is ranked the third among the product alternatives and the preference difference between product $\mathrm{A}$ and product $\mathrm{B}$ is very small. Compared with product $\mathrm{E}$ and product $\mathrm{D}$, it is direct to find that product A has smaller probabilities of target achievement in terms of customer needs $X_{1}, X_{2}$, $X_{3}$ and $X_{4}$.

The ranking of product $\mathrm{A}$ can be increased by improving $x_{1}, x_{2}, x_{3}$ or $x_{4}$. For example, after changing $x_{3}$ and $x_{4}$ into $l_{6}$ and 10 days, respectively, the purchase cost will increase to 320 Chinese Yuan and the new product alternative $A_{1}$ is designed. The calculated value of $A_{1}$ is 0.67994 . After changing $x_{1}$ and $x_{3}$ into [-0.05, 0.05] and $l_{8}$, respectively, the purchase cost will increase to 320 Chinese Yuan and the new product alternative $A_{2}$ is designed. The calculated value of $A_{2}$ is 0.71363 . 


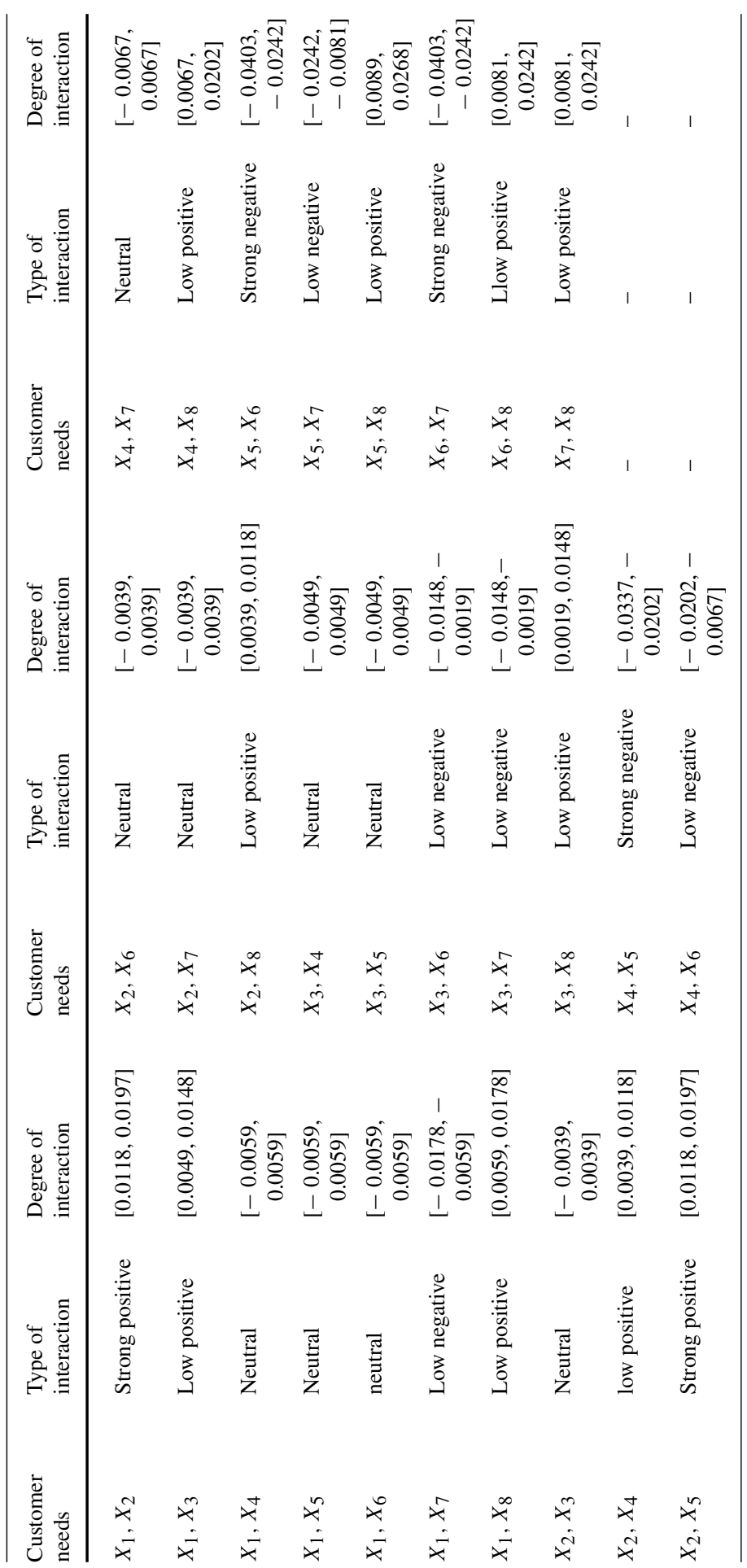




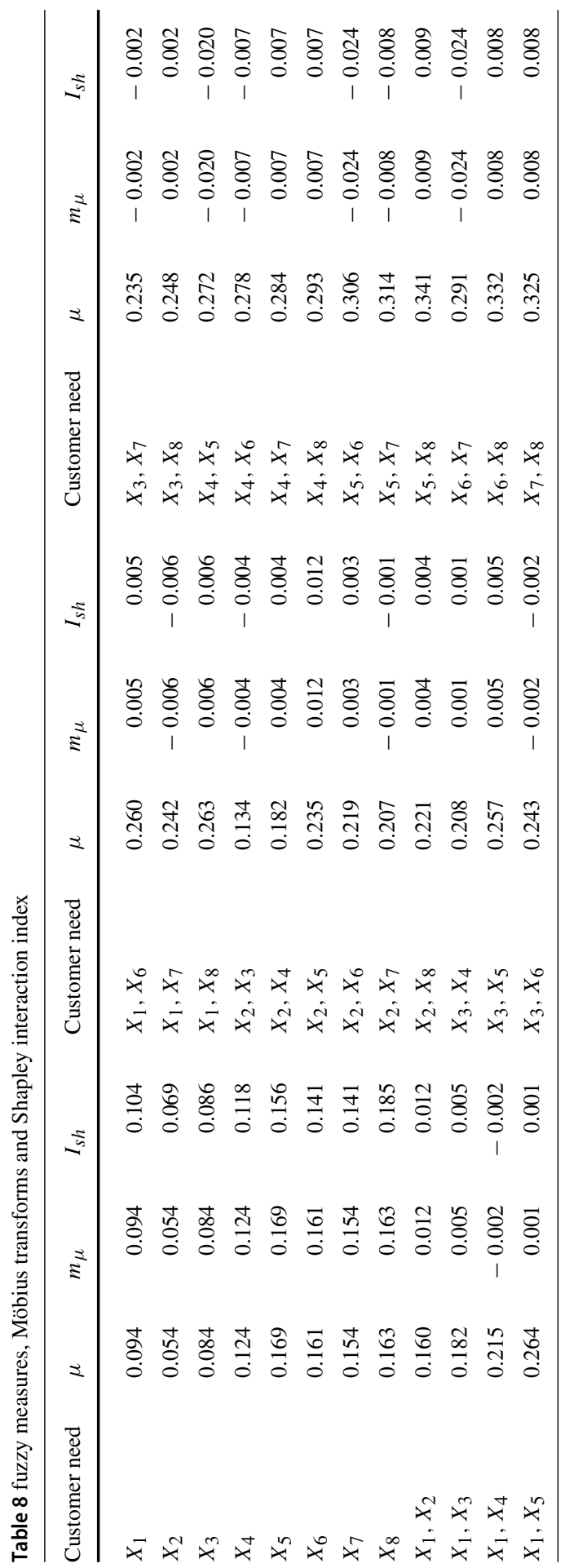




\section{Comparisons and discussions}

Two comparisons are deployed in order to identify the strengths and weaknesses of the proposed approach. One comparative analysis focuses on how to identify the coefficients of MTPFs. The other comparison focuses on different target-based approaches in terms of forms of targets and performance levels, forms of multiple attribute target achievement, and group decision-making.

\subsection{Comparative analysis in terms of identifying coefficients}

We shall use the same but more simplified example introduced in Sect. 5 to compare our approach with Keeney and Raiffa's approach (KR approach) for identifying coefficients. Suppose that each of alternatives has 3 attributes, including accuracy of measurement $\left(X_{1}\right)$, ease of use $\left(X_{3}\right)$, and purchasing cost $\left(X_{8}\right)$, and that these 3 attributes have the range as shown in Table 2.

\subsubsection{Keeney and Raiffa's approach}

To authors' knowledge, there is no available literature on identifying coefficients of MTPFs. However, according to Bordley and Kirkwood (2004), there always exist the equivalence between multilinear utility functions (MUFs) and MTPFs, which provides a feasible method to evaluate parameters of MTPFs based on MUFs. A general method for determining coefficients of additive, multiplicative and multilinear utility functions has been introduced by Keeney and Raiffa (1993). Furthermore, as shown in Sect. 2.1, the coefficients $k_{A}$ of MTPFs are decision maker's utility levels given target achievement $I$, similar with the interpretation of "corner consequence", the components of parameters in MUFs, which further enhances the rationale for using KR approach for MTPFs. Therefore, MUFs and the procedure of evaluating coefficients of MTPFs based on KR approach are demonstrated.

Given a set of attributes $X=\left\{X_{1}, X_{2}, \ldots, X_{n}\right\}$ with $n$ attributes' performance level $x=\left\{x_{1}, x_{2}, \ldots, x_{n}\right\}$, if each attribute is utility independent of its complement, then the multi-attributes utility function will reduce into a MUF:

$$
\begin{aligned}
u(x)= & \sum_{i=1}^{n} \gamma_{i} u_{i}\left(x_{i}\right)+\sum_{i=1}^{n} \sum_{j>i}^{n} \gamma_{i j} u_{i}\left(x_{i}\right) u_{j}\left(x_{j}\right)+\sum_{i=1}^{n} \sum_{j>i}^{n} \sum_{e>j}^{n} \gamma_{i j e} u_{i}\left(x_{i}\right) u_{j}\left(x_{j}\right) u_{e}\left(x_{e}\right) \\
& +\gamma_{12 \ldots n} u_{1}\left(x_{1}\right) u_{2}\left(x_{2}\right) \cdots u_{n}\left(x_{n}\right)
\end{aligned}
$$

where

1. $u(x)$ is normalized by $u\left(x_{1}^{0}, x_{2}^{0}, \ldots, x_{n}^{0}\right)=0$ and $u\left(x_{1}^{*}, x_{2}^{*}, \ldots, x_{n}^{*}\right)=1$

2. $u_{i}\left(x_{i}\right)$ is a conditional utility function over $X_{i}$

normalized by $u_{i}\left(x_{i}^{0}\right)=0$ and $u_{i}\left(x_{i}^{*}\right)=1$ 
3. The scaling constants can be expressed by

$$
\begin{aligned}
\gamma_{i}= & u\left(x_{i}^{*}, \bar{x}_{i}^{0}\right) \\
\gamma_{i j} & =u\left(x_{i}^{*}, x_{j}^{*}, \bar{x}_{i j}^{0}\right)-\gamma_{i}-\gamma_{j} \\
& =u\left(x_{i}^{*}, x_{j}^{*}, \bar{x}_{i j}^{0}\right)-u\left(x_{i}^{*}, \bar{x}_{i}^{0}\right)-u\left(x_{j}^{*}, \bar{x}_{j}^{0}\right) \\
\gamma_{i j e} & =u\left(x_{i}^{*}, x_{j}^{*}, x_{e}^{*}, \bar{x}_{i j e}^{0}\right)-\gamma_{i j}-\gamma_{i e}-\gamma_{j e}-\gamma_{i}-\gamma_{j}-\gamma_{e} \\
& =u\left(x_{i}^{*}, x_{j}^{*}, x_{e}^{*}, \bar{x}_{i j e}^{0}\right)-u\left(x_{i}^{*}, x_{j}^{*}, \bar{x}_{i j}^{0}\right)-u\left(x_{i}^{*}, x_{e}^{*}, \bar{x}_{i e}^{0}\right)-u\left(x_{j}^{*}, x_{e}^{*}, \bar{x}_{j e}^{0}\right)+u\left(x_{i}^{*}, \bar{x}_{i}^{0}\right) \\
& +u\left(x_{j}^{*}, \bar{x}_{j}^{0}\right)+u\left(x_{e}^{*}, \bar{x}_{e}^{0}\right)
\end{aligned}
$$

and, finally,

$$
\begin{aligned}
\gamma_{12 \ldots n}= & u\left(x_{1}^{*}, x_{2}^{*}, \ldots, x_{n}^{*}\right)-\sum_{i=1}^{n} u\left(x_{i}^{0}, \bar{x}_{i}^{*}\right)+\cdots+(-1)^{n-2} \sum_{i=1, j>i}^{n} u\left(x_{i}^{*}, x_{j}^{*}, \bar{x}_{i j}^{0}\right) \\
& +(-1)^{n-1} \sum_{i=1}^{n} u\left(x_{i}^{*}, \bar{x}_{i}^{0}\right)
\end{aligned}
$$

Obviously, there are $2^{n}-1$ parameters in the MUF, the same as MTPFs. There is also similarity in the interpretation of some special utilities between the MTPF and MUF. For example, with three attributes, $u\left(x_{1}^{*}, x_{2}^{0}, x_{3}^{0}\right)$ represents the utility of decision maker for consequence where $x_{1}$ is at most preferred preference and both of $x_{2}$ and $x_{3}$ are at their least preferred preference, while $k_{1}=u(1,0,0)$ denotes decision maker's utility when $x_{1}$ achieves its target and both of $x_{2}$ and $x_{3}$ fail to achieve their targets. The similarity establishes relationships between $k_{A}$ and $\gamma$. On the premise of equivalence between MUFs and MTPFs, considering utility explanation of the latter, it's straightforward to transform the evaluation of coefficients in MTPFs into the evaluation of scaling constants in MUFs.

Once the $u_{i}^{\prime} s$ have been assessed, KR approach suggests the evaluation of $2^{n}-1$ parameters depend on a set of $2^{n}-1$ independent equations. The set of equations can be generated from certainty considerations, probabilistic considerations, or combination of both. We will apply KR approach to the above simplified example.

According to Eq. (2), we can get a MTPF with 3 attributes:

$$
\begin{aligned}
u_{M L}(x)= & k_{1} p_{1}\left(1-p_{3}\right)\left(1-p_{8}\right)+k_{3} p_{3}\left(1-p_{1}\right)\left(1-p_{8}\right)+k_{8} p_{8}\left(1-p_{1}\right)\left(1-p_{3}\right) \\
& +k_{13} p_{1} p_{3}\left(1-p_{8}\right)+k_{18} p_{1} p_{8}\left(1-p_{3}\right) \\
& +k_{38} p_{3} p_{8}\left(1-p_{1}\right)+k_{138} p_{1} p_{3} p_{8}
\end{aligned}
$$

where $k_{A=\left\{i \mid I_{i}=1\right\}}=u\left(I_{i}^{1}, \bar{I}_{i}^{0}\right)$. Subject to Bordley's theorem of equivalence between MTPFs and MUFs, $k_{A}=u\left(I_{i}^{1}, \bar{I}_{i}^{0}\right)=u\left(x_{i}^{*}, \bar{x}_{i}^{0}\right)$, and (48) can be converted into

$$
u_{M L}(x)=\gamma_{1} p_{1}+\gamma_{3} p_{3}+\gamma_{8} p_{8}+\gamma_{13} p_{1} p_{3}+\gamma_{18} p_{1} p_{3}+\gamma_{38} p_{3} p_{8}+\gamma_{138} p_{1} p_{3} p_{8}
$$


where

$$
\begin{aligned}
\gamma_{i}= & k_{i}=u\left(x_{i}^{*}, \bar{x}_{i}^{0}\right) \\
\gamma_{i j}= & k_{i j}-k_{i}-k_{j}=u\left(x_{i}^{*}, x_{j}^{*}, \bar{x}_{i j}^{0}\right)-u\left(x_{i}^{*}, \bar{x}_{i}^{0}\right)-u\left(x_{j}^{*}, \bar{x}_{j}^{0}\right) \\
\gamma_{i j e}= & k_{i j e}-k_{i j}-k_{i e}-k_{j e}+k_{i}+k_{j}+k_{e} \\
= & u\left(x_{i}^{*}, x_{j}^{*}, x_{e}^{*}\right)-u\left(x_{i}^{*}, x_{j}^{*}, \bar{x}_{i j}^{0}\right)-u\left(x_{i}^{*}, x_{e}^{*}, \bar{x}_{i e}^{0}\right)-u\left(x_{j}^{*}, x_{e}^{*}, \bar{x}_{j e}^{0}\right)+u\left(x_{i}^{*}, \bar{x}_{i}^{0}\right) \\
& +u\left(x_{j}^{*}, \bar{x}_{j}^{0}\right)+u\left(x_{e}^{*}, \bar{x}_{e}^{0}\right)
\end{aligned}
$$

After $p_{i}^{\prime} s$ have been assessed, the key to solve MTPFs is to evaluate the 7 parameters and the evaluation of $k_{A}$ is transformed into assessing $\gamma$. Additionally, the least preferred and most preferred levels of $x_{i}^{\prime} s$ for products are shown in Table 2.

Then, the following steps are used to assess $\gamma$ for the case.

\section{Step 1: Ranking $\gamma_{i}$ 's}

The first step is to order the $\gamma_{i}$ 's. This can be done by asking the decision maker whether she/he prefers $u\left(x_{i}^{*}, \bar{x}_{i}^{0}\right)$ to $u\left(x_{j}^{*}, \bar{x}_{j}^{0}\right)$. According to Eq. (49-1), if the former is preferred, then $\gamma_{i}>\gamma_{j}$; if the latter is preferred, then $\gamma_{j}>\gamma_{i}$; and if they are equally preferable, the $\gamma_{i}=\gamma_{j}$. Repeating this for all binary comparisons, at most $\left(n^{2}-n\right) / 2$ times, a complete ranking of the $\gamma_{i}^{\prime} s$ will be elicited. In order to help the decision maker to make better assessments, questions can be set up to be more comprehensible, for instance, "imagine that each of the performance measures are at the state $x_{i}^{0}$, would you rather have attribute $\mathrm{X}_{1}$ pushed to $x_{1}^{*}$ than $\mathrm{X}_{3}$ pushed to $x_{3}^{*}$ ?". Finally, we ascertain $\gamma_{8}>\gamma_{1}>\gamma_{3}$.

\section{Step 2: Evaluating the largest $\gamma_{i}$}

The next step is to assess the largest scaling constant $\gamma_{8}$ using probabilistic question. Question A. For what probability $p^{\prime}$ is decision maker indifferent between:

1. The lottery giving a $p^{\prime}$ chance at $x^{*}$ and a $1-p^{\prime}$ chance at $x^{0}$.

2. And the consequence $\left(x_{1}^{0}, x_{3}^{0}, x_{8}^{*}\right)$.

The decision maker's answer is $p_{8}^{\prime}=0.2$, then we get

$$
\gamma_{8}=p_{8}^{\prime}
$$

\section{Step 3: Evaluating the other $\lambda_{j}$ 's}

The third step is to evaluate the magnitude of the other $\gamma_{j}$ 's relative to the largest $\gamma_{8}$ using the certainty question.

Question B. Select a level of target achievement $X_{i}\left(\right.$ e.g., $\left.x_{i}^{\prime}\right)$ and a level of $X_{j}\left(\right.$ e.g., $\left.x_{j}^{\prime}\right)$ so that, for any fixed levels of all other attributes, decision maker is indifferent between:

1. A consequence yielding $x_{i}^{\prime}$ and $x_{j}^{0}$ together.

2. And a consequence yielding $x_{j}^{\prime}$ and $x_{i}^{0}$ together. 
The decision maker is required to choose a level of $x_{8}$ in $\left[x_{8}^{0}, x_{8}^{*}\right]$, call it $x_{8}^{\prime}$, so that $\left(x_{1}^{0}, x_{3}^{0}, x_{8}^{\prime}\right)$ and $\left(x_{1}^{*}, x_{3}^{0}, x_{8}^{0}\right)$ are equally preferable. From Eqs. (47-2) and (49), it follows that

$$
k_{8} u_{8}\left(x_{8}^{\prime}\right)=\gamma_{1}
$$

Similarly, the decision maker is asked for another $x_{8}^{\prime \prime}$ so that $\left(x_{1}^{0}, x_{3}^{0}, x_{8}^{\prime \prime}\right) \sim$ $\left(x_{1}^{0}, x_{3}^{*}, x_{8}^{0}\right)$ to yield

$$
k_{8} u_{8}\left(x_{8}^{\prime \prime}\right)=\gamma_{3}
$$

where $u_{8}\left(x_{8}^{\prime}\right)$ and $u_{8}\left(x_{8}^{\prime \prime}\right)$ have been assessed.

\section{Step 4: Evaluating all $\gamma_{i j}{ }^{\prime}$ s}

The assessment of the $\gamma_{i j}^{\prime} s$ is no more different from the assessment of $\gamma_{i}^{\prime} s$. According to Eq. (49-2), the values of $\gamma_{i j}^{\prime} s$ can be clearly generated using the "corner consequence" $u\left(x_{i}^{*}, x_{j}^{*}, \bar{x}_{i j}^{0}\right)$. However, what is evaluated in practice is the relative value of the other $\gamma_{i e}$ 's with respect to some $\gamma_{i j}$. For instance, the decision maker is asked: "What is the value of $p_{18}^{\prime}$ so that you are indifferent between $\left\langle x_{1}^{*}, x_{3}^{0}, x_{8}^{*}\right\rangle$ and $\left\langle\left(x_{1}^{*}, x_{3}^{*}, x_{8}^{*}\right), p_{18}^{\prime},\left(x_{1}^{0}, x_{3}^{0}, x_{8}^{0}\right)\right)$ ?" If the decision maker's response is 0.3 , then $u\left(x_{1}^{*}, x_{3}^{0}, x_{8}^{*}\right)=0.3$, from Eq. (49-2), it follows that

$$
\gamma_{18}=u\left(x_{1}^{*}, x_{3}^{0}, x_{8}^{*}\right)-\gamma_{1}-\gamma_{8}=0.3-\gamma_{1}-\gamma_{8}
$$

Proceeding with the questions "What is the value of $p_{13}^{\prime}$ so that the decision maker is indifferent between $\left\langle x_{1}^{*}, x_{3}^{0}, x_{8}^{*}\right\rangle$ and $\left\langle\left(x_{1}^{*}, x_{3}^{*}, x_{8}^{0}\right), p_{13}^{\prime},\left(x_{1}^{0}, x_{3}^{0}, x_{8}^{0}\right)\right)$ ?" and "What is the value of $p_{38}^{\prime}$ so that decision maker is indifferent between $\left\langle x_{1}^{*}, x_{3}^{0}, x_{8}^{*}\right\rangle$ and $\left\langle\left(x_{1}^{0}, x_{3}^{*}, x_{8}^{*}\right), p_{38}^{\prime},\left(x_{1}^{0}, x_{3}^{0}, x_{8}^{0}\right)\right)$ ?" If the answers are 0.8 and 0.7 respectively, from (47-1), (47-2) and (49), it follows that

$$
\begin{aligned}
& \gamma_{1}+\gamma_{8}+\gamma_{18}=0.8\left(\gamma_{1}+\gamma_{3}+\gamma_{13}\right) \\
& \gamma_{1}+\gamma_{8}+\gamma_{18}=0.7\left(\gamma_{3}+\gamma_{8}+\gamma_{38}\right)
\end{aligned}
$$

\section{Step 5: Evaluating $\gamma_{i j e}$}

The sum of all scaling constants is one. Thus, the final equation can be obtained as follows:

$$
\gamma_{1}+\gamma_{3}+\gamma_{8}+\gamma_{13}+\gamma_{18}+\gamma_{38}+\gamma_{138}=1
$$




\section{Step 6: Solving all the identified equations}

Suppose that $u_{8}\left(x_{8}^{\prime}\right)$ and $u_{8}\left(x_{8}^{\prime \prime}\right)$ has been assessed with values of 0.8 and 0.5 , respectively, then Eqs. (50-1-50-7) can be solved for all of $\gamma_{i}$ 's, $\gamma_{i j}$ 's and $\gamma_{i j e}$. The results are:

$$
\gamma_{1}=0.16, \gamma_{3}=0.1, \gamma_{8}=0.2, \gamma_{13}=0.115, \gamma_{18}=-0.06, \gamma_{38}=0.129, \gamma_{138}=0.356
$$

Finally, the MUF of this case is obtained as follows:

$$
u_{M L}(x)=0.16 p_{1}+0.1 p_{3}+0.2 p_{8}+0.115 p_{1} p_{3}-0.06 p_{1} p_{8}+0.129 p_{3} p_{8}+0.356 p_{1} p_{3} p_{8}
$$

In the light of the above function, evaluation and rank of different products can be accomplished.

\subsubsection{Comparative analysis of two approaches for determining coefficients}

Comparatively, the proposed approach is different from the KR approach in terms of evaluation strain, independence check, consistency check and parameters simplification, as summarized in Table 9.

Evaluation strain The evaluation of parameters using KR approach is not a negligible burden on analysts. The essence of establishing the sets of equations, whether based on certainty or probabilistic consideration, is to obtain the indifferent probabilities to compare two or more different attributes' performance levels $x$ 's. Throughout the process, a decision maker has to spend a great deal of time in intense and thoughtful thinking, and it is the mentally heavy burden. Furthermore, the impact of varying multiple attributes simultaneously on utility when the number of attributes increase need to be taken into account, which is another challenge for evaluation.

In contrast, our approach derives coefficients through some rigorous mathematical methods, and analyst is merely asked to provide the degree of interactions between an arbitrary pair of attributes, which is moderate for decision maker. It exerts less mental pressure on evaluators and is easier operationally than KR approach.

Independence check In KR approach, one operational problem of concern is how to avoid redundancy and guarantee independence among equations. In step 3, suppose that decision maker is asked for $x_{8}^{\prime \prime \prime}$ so that $\left(x_{1}^{*}, x_{3}^{0}, x_{8}^{\prime \prime \prime}\right) \sim\left(x_{1}^{*}, x_{3}^{*}, x_{8}^{0}\right)$ where the

Table 9 Comparative analysis of two approaches for determining coefficients

\begin{tabular}{lllll}
\hline Approach & Evaluation strain & Independence check & Consistency check & $\begin{array}{l}\text { Parameters } \\
\text { simplification }\end{array}$ \\
\hline KR approach & Strong & Yes & Require more & No \\
Our approach & Moderate & No & Require less & Yes \\
\hline
\end{tabular}


fixed level of $x_{1}$ is $x_{1}^{*}$ instead of $x_{1}^{0}$, then equating expected utilities,

$$
\gamma_{1}+\gamma_{8} u_{8}^{\prime \prime \prime}+\gamma_{18} u_{8}^{\prime \prime \prime}=\gamma_{1}+\gamma_{3}+\gamma_{13}
$$

where $u_{8}^{\prime \prime \prime}$ is known. Clearly, Eqs. (50-1-50-2) and Eqs. (50-4-50-8) are seven equations with seven unknows, but Eq. (50-6) and Eq. (50-8) are not independent of each other. Both are establishing the connections between $\left(\gamma_{1}, \gamma_{8}, \gamma_{18}\right)$ and $\left(\gamma_{1}, \gamma_{3}, \gamma_{13}\right)$, failing to determine all scaling constants. Keeney reckons that the understanding of the problem and knowledge of the functional form of the utility function are probably the best guards against redundant equations. It means that we have to check independence of equations repeatedly during the solution process.

However, our approach constructs a solution where the determination of coefficients relies on objective function focusing only on the importance and interactions of attributes, and is not troubled by independence check.

Consistency check Since KR approach is a kind of subjective assessment method, there may be deviation of the results from the actual preference of decision maker. For instance, in step 1, when questions are substituted for "imagine that each of the performance measures are at the state $x_{i}^{*}$, would you rather have attribute $\mathrm{X}_{3}$ and $\mathrm{X}_{8}$ lowered to $x_{3}^{0}$ and $x_{8}^{0}$ than $\mathrm{X}_{1}$ and $\mathrm{X}_{8}$ lowered to $x_{1}^{0}$ and $x_{8}^{0}$ ?", it might turn out that $\gamma_{1}<\gamma_{3}$ instead $\gamma_{1}>\gamma_{3}$. In this case, decision maker cannot avoid thinking hard about the issue and modify some of the assumptions or evaluations in order to attain consistency, which is tedious and time consuming. Furthermore, sensitivity analysis should be taken into account to determine whether the inconsistency is worth resolving.

In our approach, inconsistency may result from erroneously appraising the relationships between two attributes, knowledge of which is the basis of identifying either Banzhaf importance index or Banzhaf interaction index. For example, $\phi_{B}(1)>\phi_{B}(8)$ maybe substituted for $\phi_{B}(1)<\phi_{B}(8)$ when direct-relation matrix varies in Table 5, which has the effect on the values of $I_{B}(i, j)^{\prime} s$ and further shifts the ranking of alternatives. It is desirable that the assessed function faithfully represents decision maker's preferences, and it seems that our approach and the KR approach can be used to validate each other's consistencies. Intuitively, inconsistency is more likely to occur through the KR approach.

Parameter simplification Taking the KR approach, coefficients in MTPFs can be regarded as indicators of the importance of their relative attributes, also known as scaling constants, and are combinations of some special utility levels. During the identification process, there are always $2^{n}-1$ parameters to be determined. With an increase of $n$, the computing burden placed on the decision maker by KR approach increases very quickly. Thus, extant literature averts the problem by assessing stronger preference assumptions than utility independence and simplifying multilinear forms into additive or multiplicative forms.

Compared to the KR approach, we interpret parameters as Möbius transformation of fuzzy measures, representing the importance of the set of corresponding target achievement probabilities. Under the context of fuzzy measures, we are allowed to simplify MTPFs by using different fuzzy measures to reduce the number of parameters. 
For example, the 2-additive fuzzy measures only care about interactions between every pair of attributes, i.e., $m_{\mu}(S)_{|S| \geq 3}=0$. Hence, the number of coefficients to be determined is reduced to $n(n+1) / 2$. As $n$ increases, the advantage will be more pronounced. For instance, with only three attributes, the KR approach only needs to evaluate one more coefficient than our method. However, for $n=7$ the difference rapidly becomes 100 .

\subsection{Comparative analysis of different target-based approaches}

The proposed approach is compared with the other 4 approaches of TBDA in terms of forms of targets and performance levels, aggregation functions of multiple attribute target achievement and group decision making (Table 10).

The forms of targets and performance levels in the seminal paper by Bordley and Kirkwood (2004) are mainly probability distributions, assuming that there are possibly uncertain targets and performance levels in the problems of resource allocation under uncertain competition. Yan et al. integrate fuzzy set theory into TBDA and introduce three typical types of fuzzy targets. To solve the strategic freight forwarder selection of China Southern Airlines, the forms of targets and performance levels are crisp numbers, simple linguistic terms, ULTs, considering the characteristics of decision attributes and knowledge of experts. Wang et al. further extends the range of linguistic expression of targets and performance levels. Complex linguistic expressions, including ULTs, HFLTSs, LTWHs are involved to express the uncertainties in the linguistic setting. Based on the above contributions, these different forms of expressions, including crisp numbers, interval numbers, fuzzy numbers, probability distributions, simple linguistic terms and complex linguistic expressions are synthesized into the proposed approach. These diverse forms except crisp numbers are then transformed into probability distributions for measuring the probability of target achievement.

Regarding to aggregation functions of multiple attribute target achievement, Bordley and Kirkwood (2004) lays the foundation of three types of target-based preference

Table 10 Comparison of different target-based approaches

\begin{tabular}{|c|c|c|c|}
\hline Reference & $\begin{array}{l}\text { Forms of targets and } \\
\text { performance levels }\end{array}$ & Aggregation functions & $\begin{array}{l}\text { Group decision } \\
\text { making }\end{array}$ \\
\hline $\begin{array}{l}\text { Bordley and Kirkwood } \\
\text { (2004) }\end{array}$ & Probability distributions & $\begin{array}{l}\text { Additive form, } \\
\text { multiplicative form, } \\
\text { multilinear form }\end{array}$ & No \\
\hline Yan et al. (2013) & Fuzzy numbers & $\begin{array}{l}\text { Choquet integral and } \\
\text { fuzzy measures }\end{array}$ & No \\
\hline Feng and Lai (2014) & $\begin{array}{l}\text { Crisp numbers, simple } \\
\text { linguistic terms, ULTs }\end{array}$ & Weighted TOPSIS & Yes \\
\hline Wang et al. (2019) & ULTs, HFLTSs, LTWHs & Additive form & Yes \\
\hline The proposed approach & $\begin{array}{l}\text { Synthesis of the above } \\
\text { forms }\end{array}$ & Multilinear form & No \\
\hline
\end{tabular}


functions, including additive form, multiplicative form and multilinear form. These three types of preference functions imply additive target preferences, reliabilitystructured target preferences and independent target, respectively. However, the application of target-based preference functions focuses on additive form and there is no available method to identify coefficients of MTPF. Yan et al. introduces Choquet integral and fuzzy measures to aggregate non-additively multiple attribute target achievement, which implies a different preference assumption among targets. From a different perspective, Feng and Lai utilizes the weighted TOPSIS to rank alternatives based on the distance of each alternative from best and worst alternatives. The additive form is used in Feng and Lai (2014) to aggregate multiple attribute target achievement, which implies the assumption of additive target preferences being verified. Under a weaker assumption of independent target, the proposed approach focuses on MTPFs, presenting a novel procedure to identify the coefficients of MTPFs based on the multilinear model and 2-additive fuzzy measures.

Group decision-making is incorporated into TBDA in Feng and Lai (2014); Wang et al., 2019). However, their mechanisms are different. Individual rankings of alternatives are aggregated into a group ranking in Feng and Lai (2014) while individual assessments of single attribute target achievement are aggregated into a group assessment of single attribute target achievement in Wang et al. (2019). Comparatively, there are no explicit consideration of group decision-making in the other approaches, and they are suitable for the cases of individual decision-making or group decision-making with group consensus.

\subsection{Discussions}

For several classes of decisions, such as product development, regulated environments and setting performance standards, TBDA is more natural approach compared to the utility analysis (Bordley \& Kirkwood, 2004). Recent progress on TBDA mainly focuses on different expression forms of targets and performance levels, and on different types of aggregation functions of multiple attribute target achievement.

Theoretically, this study focuses on these two aspects and contributes to proposing a novel approach for multilinear hybrid-information TBDA. First, considering that different forms of expression of targets and performance levels are usually introduced separately in literature, crisp numbers, interval numbers, fuzzy numbers, probability distributions, simple linguistic terms, and complex linguistic expressions are incorporated into the proposed approach. These different forms are then transformed into probability distributions to enable calculating probability of single attribute target achievement. Therefore, a more inclusive framework is given to allow diverse forms of targets and performance levels. The second and a more important contribution is the novel procedure to identify the coefficients of MTPFs. To the best of our knowledge, although the KR approach is used to identify the coefficients of multi-attribute utility functions, there is no other method for identifying the coefficients of MTPFs so far. The proposed procedure to identify the coefficients of MTPFs is enabled by the established equivalence between MTPFs and the multilinear model with respect to fuzzy measures. Considering the characteristics of the multilinear model, the Banzhaf 
importance index and the Banzhaf interaction index are used to describe the multilinear model and MTPFs. 2-additive fuzzy measures are also introduced. MTPFs are then expressed by the Banzhaf importance index for each attribute and the Banzhaf interaction index for any pair of attributes. Therefore, the proposed approach is able to identify the coefficients of MTPFs in a 2-additive way.

Practically, considering the inherent complexity of problems along with different perception and knowledge levels of decision makers, diverse assessments using crisp numbers, interval numbers, fuzzy numbers, probability distributions, simple linguistic terms, and complex linguistic expressions allow fruitful and flexible assessments of targets and performance levels. Also, compared with the KR approach in terms of identifying coefficients, the proposed approach has certain advantages in terms of evaluation strain, independence check, and consistency check, which can balance between complexity and accuracy of identifying coefficients.

To apply the proposed approach in practical problems, two assumptions should be verified, including independent target and probability independence among attributes. The assumption of independent target is required for MTPFs, which is a weaker assumption than additive target preferences in additive form of target-based preference functions, and it seems intuitively reasonable for many target-oriented decisions (Bordley \& Kirkwood, 2004). Probability independence is necessary to calculate the multilinear target-based expected utility function in Eq. (13). If these two assumptions are valid, then it is straightforward to use the four-step procedure in Sect. 4.4 to support multilinear hybrid-information TBDA. According to Bordley and Kirkwood (2004), the additive form of target-based preference functions is a special case of MTPFs. Therefore, the proposed approach can be applied in a wider scale than that of additive form in practical problems.

The proposed approach suffers from two limitations which could be improved in the future. First, there are cases that independent target cannot be verified, which makes MTPFs fail to model these kinds of situations. Second, group consensus is implicit in the proposed approach, which may not be achievable in case of calculating probabilities of target achievement and identifying coefficients in MTPFs. If diverse assessments among the group of decision makers are obvious, group decision-making should be further incorporated into the proposed approach, otherwise the quality of decision results can degrade (Lin \& Zhan \& Xu 2020).

\section{Conclusions}

In this paper, the multilinear hybrid-information TBDA approach is proposed. The main scientific contributions of the proposed approach are twofold. First, the approach allows the decision maker to assess fruitful and flexible forms of targets and performance levels to enable effective evaluation, considering the inherent complexity of practical problems and different levels of cognition and knowledge of the decision maker. It can manipulate different expression forms of targets and performance levels simultaneously by transforming different expression forms uniformly into probability distributions except the ones with the form of crisp numbers. The calculation of probability of target achievement is then conducted for four types of comparisons 
in terms of expression forms. Second, the MTPF is transformed into the multilinear model with respect to fuzzy measures on basis of the established equivalence between them. Therefore, the coefficient identification of MTPFs is turned into that of the multilinear model based on fuzzy measures. In view of the characteristics of the multilinear model, the Banzhaf importance index and the Banzhaf interaction index are introduced to describe the multilinear model rather than the Shapley importance index and the Shapley interaction index. In order to reduce the complexity of identifying coefficients in the multilinear model, 2-additive fuzzy measures are introduced and the multilinear model is then expressed by the Banzhaf importance index for each attribute and the Banzhaf interaction index for any pair of attributes. In particular, the Banzhaf importance index is determined using DEMATEL method while 2-additive fuzzy measures and the Banzhaf interaction index are identified through minimum variance method. The approach is thus able to deal with diverse assessment forms of targets and performance levels simultaneously and provides a novel way to model and solve MTPFs. The approach is applied for customer competitive evaluation of smart thermometer patches. The application shows that the approach enables effective multilinear hybrid-information TBDA.

In terms of future research, two research directions are identified. First, it would be useful to consider copulas and copula-based extensions of fuzzy measures in TBDA (Spizzichino \& Fabio, 2019), which has the potential to model probability dependence and dependent target preferences. This will extend target-based formations into more general situations. Second, as group consensus is assumed in the proposed approach, it would be interesting to incorporate diverse group assessments in calculating probabilities of target achievement and solving coefficients in MTPFs.

Acknowledgements The authors appreciate the editor and anonymous reviewers for their helpful comments on this paper.

Author contributions Conceptualization: XZ, ST; Methodology: XZ, QF, ST; Data curation: XZ; Writing—original draft: XZ, QF; Writing_review \& editing: XZ, QF, HE; Validation: HE.

Funding The research leading to these results received funding from the Humanity and Social Science Foundation of Ministry of Education of China under Grant Agreement No [19YJA630119]; and the Natural Science Basic Research Program of Shaanxi under Grant Agreement No [2021JM-077].

\section{Declarations}

Conflict of interest The authors have no conflicts of interest to declare that are relevant to the content of this article.

Consent for publication Written informed consent for publication was obtained from all participants.

\section{References}

Beliakov, G., James, S., \& Wu, J. Z. (2020). Discrete fuzzy measures: computational aspects. Springer. Berhold, M. H. (1973). The use of distribution functions to represent utility functions. Management Science, 19, 825-829. https://doi.org/10.1287/mnsc.19.7.825 
Borch, K. (1968). Decision rules depending on the probability of ruin. Oxford Economic Papers, 20, 1-10. https://doi.org/10.1287/opre.1030.0093

Bordley, R. F., \& Kirkwood, C. W. (2004). Multiattribute preference analysis with performance targets. Operations Research, 52(6), 823-835. https://doi.org/10.1287/opre.1030.0093

Bordley, R. F., \& Licalzi, M. (2000). Decision analysis using targets instead of utility functions. Decisions in Economics and Finance, 23, 53-74. https://doi.org/10.1007/s102030050005

Castagnoli, E., \& Licalzi, M. (1996). Expected utility without utility. Theory and Decision, 41, $281-301$. https://doi.org/10.1007/BF00136129

Feng, B., \& Lai, F. J. (2014). Multi-attribute group decision making with aspirations: A case study. OmegaInternational Journal of Management Science, 44, 136-147. https://doi.org/10.1016/j.omega.2013. 07.003

Grabisch, M. (2016). Set Functions, Games and Capacities in Decision Making. Springer.

Huynh, V. N., Nakamori, Y., Ryoke, M., \& Ho, T. B. (2007). Decision making under uncertainty with fuzzy targets. Fuzzy Optimization and Decision Making, 6(3), 255-278. https://doi.org/10.1007/s10700007-9011-0

Huynh, V. N., Yan, H. B., \& Nakamori, Y. (2010). A target-based decision-making approach to consumer-oriented evaluation model for Japanese traditional crafts. IEEE Transactions on Engineering Management, 57(4), 575-588. https://doi.org/10.1109/TEM.2009.2025494

Keeney, R. L., \& Raiffa, H. (1993). Decisions with multiple objectives: Preferences and value tradeoffs New York. Cambridge University Press.

Kosanoglu, F., \& Bierb, M. (2020). Target-oriented utility for interdiction of transportation networks. Reliability Engineering \& System Safety, 197, 106793. https://doi.org/10.1016/j.ress.2020.106793

Lin, M., Chen, Z., Chen, R., \& Fujita, H. (2021a). Evaluation of startup companies using multicriteria decision making based on hesitant fuzzy linguistic information envelopment analysis models. International Journal of Intelligent Systems, 7(1), 11195-11208. https://doi.org/10.1002/int.22379

Lin, M., Huang, C., Chen, R., Fujita, H., \& Wang, X. (2021b). Directional correlation coefficient measures for Pythagorean fuzzy sets: Their applications to medical diagnosis and cluster analysis. Complex Intelligent Systems, 7, 1025-1043. https://doi.org/10.1007/s40747-020-00261-1

Lin, M., Wang, H., \& Xu, Z. (2020). TODIM-based multi-criteria decision-making method with hesitant fuzzy linguistic term sets. Artificial Intelligence Review, 53(4), 3647-3671. https://doi.org/10.1007/ s10462-019-09774-9

Lin, M., Zhan, Q., \& Xu, Z. (2020). Decision making with probabilistic hesitant fuzzy information based on multiplicative consistency. International Journal of Intelligent Systems, 35(8), 1233-1261. https:// doi.org/10.1002/int.22240

Spizzichino, F. L. (2019). On the probabilistic meaning of copula-based extensions of fuzzy measures. Applications to target-based utilities and multi-state reliability systems. Fuzzy Sets Systems, 354, 1-19. https://doi.org/10.1016/j.cie.2017.06.020

Tsetlin, I., \& Winkler, R. L. (2006). On equivalent target-oriented formulations for multiattribute utility. Decision Analysis, 3(3), 94-99. https://doi.org/10.1287/deca.1060.0068

Tsetlin, I., \& Winkler, R. L. (2007). Decision making with multiattribute performance targets: The impact of changes in performance and target distributions. Operations Research, 55(2), 226-233. https://doi. org/10.1287/opre.1060.0343

Wang, H., Xu, C., Xu, Z. S., Zeng, X. J., \& Pedrycz, W. (2019). An aspiration-based approach for qualitative decision-making with complex linguistic expressions. IEEE Access, 7, 12529-12546. https://doi.org/ 10.1109/ACCESS.2019.2892844

Wang, H., Xu, Z., \& Zeng, X. (2017). Hesitant fuzzy linguistic term sets for linguistic decision making: Current developments, issues and challenges. Information Fusion, 43, 1-12. https://doi.org/10.1016/ j.inffus.2017.11.010

Wang, H., Xu, Z., \& Zeng, X. (2018). Linguistic terms with weakened hedges: A model for qualitative decision making under uncertainty. Information Sciences an International Journal, 433, 37-54. https:// doi.org/10.1016/j.ins.2017.12.036

Wu, J. Z., \& Zhang, Q. (2010). 2-order additive fuzzy measure identification method based on diamond pairwise comparison and maximum entropy principle. Fuzzy Optimization and Decision Making, 9(4), 435-453. https://doi.org/10.1007/s10700-010-9086-х

Yan, H. B., Huynh, V. N., Ma, T., \& Nakamori, Y. (2013). Non-additive multi-attribute fuzzy target-oriented decision analysis. Information Sciences, 240, 21-44. https://doi.org/10.1016/j.ins.2013.03.050 
Yan, H. B., Huynh, V. N., Murai, T., \& Nakamori, Y. (2008). Kansei evaluation based on prioritized multiattribute fuzzy target-oriented decision analysis. Information Sciences, 178(21), 4080-4093. https:// doi.org/10.1016/j.ins.2008.06.023

Publisher's Note Springer Nature remains neutral with regard to jurisdictional claims in published maps and institutional affiliations. 